doi: 10.31236/osf.io/742nv

\title{
Understanding ratio of forces during early acceleration: calculation considerations and implications for practice
}

Neil Bezodis ${ }^{1}$, Steffi Colyer ${ }^{2}$, Ryu Nagahara ${ }^{3}$, Helen Bayne ${ }^{4}$, Ian Bezodis ${ }^{5}$, Jean-Benoît Morin ${ }^{6}$, Munenori Murata ${ }^{3}$, Pierre Samozino ${ }^{7}$

\footnotetext{
${ }^{1}$ Applied Sports, Technology, Exercise and Medicine Research Centre, Swansea University, Bay Campus, Crymlyn Burrows, SA1 8EN, UK. ORCID: 0000-0003-2229-3310. Twitter: @neilbezodis

2 Department for Health, University of Bath, Bath, UK. ORCID: 0000-0002-4973-6591. Twitter: @SteffiColyer

${ }^{3}$ National Institute of Fitness and Sports in Kanoya, 1 Shiromizu-cho, Kanoya, Kagoshima 891-2393, Japan. Nagahara ORCID: 0000-0001-9101-9759. Nagahara Twitter:

@R_Nagahara

${ }^{4}$ Department of Physiology, Faculty of Health Sciences, University of Pretoria, South Africa. ORCID: 0000-0002-2520-4937. Twitter: @HelenBayneZA

${ }^{5}$ Cardiff School of Sport \& Health Sciences, Cardiff Metropolitan University, UK. ORCID: 0000-0002-0250-032X. Twitter: @lanBez

${ }^{6}$ UJM-Saint-Etienne Interuniversity Laboratory of Human Movement Biology, EA 7424, University of Lyon, Saint-Étienne, France. ORCID: 0000-0003-3808-6762. Twitter:

@jb_morin

7 Univ Savoie Mont Blanc, Laboratoire Interuniversitaire de Biologie de la Motricité, EA 7424, F-73000 Chambéry, France. ORCID : 0000-0002-1665-870X. Twitter: @PierreSamozino.
}

Corresponding Author: Neil Bezodis (n.e.bezodis@swansea.ac.uk). All authors have read and approved this version of the manuscript. This article was last modified on $18^{\text {th }}$ September 2020.

Supplementary information (video file) available at: https://osf.io/n8wmf/

Please cite as Bezodis, N., Colyer, S., Nagahara, R., Bayne, H., Bezodis, I., Morin, J.-B., Murata, M. \& Samozino, P. (2020). Understanding ratio of forces during early acceleration: calculation considerations and implications for practice. SportRXiv.

https://doi.org/10.31236/osf.io/742nv 


\section{Abstract}

This study aimed to: 1) understand how the calculation of step-averaged ratio of forces (RF; horizontal component as a percentage of total ground reaction force (GRF) magnitude) affects the RF-horizontal velocity $\left(\mathrm{v}_{\mathrm{H}}\right)$ profile; 2$)$ establish the importance of RF to early acceleration (i.e. up to the end of step 4) performance; 3) assess how well the RF- $\mathrm{v}_{\mathrm{H}}$ profile obtained from a simple macroscopic model over the entire acceleration phase corresponds to the true external kinetics during just early acceleration. Twenty-four trained male sprinters completed four maximal effort $60 \mathrm{~m}$ sprints (two standing starts, two block starts), and GRFs from the block exit/initial push off and first $50 \mathrm{~m}$ on the track were analysed. The use of step-averaged force data to obtain step-averaged RF consistently led to a greater (systematic bias: +8.6 to $+9.2 \%$ ) theoretical maximal $\mathrm{RF}\left(\mathrm{RF}_{0}\right)$ and a steeper (systematic bias $=-1.7 \% \cdot \mathrm{s} / \mathrm{m}$ ) rate of decline in $R F\left(D_{R F}\right)$ than the use of instantaneous RF data, and the use of step-averaged force data was proposed as yielding values with a more appropriate assessment of the "mechanical effectiveness" over the entire step. Average RF during early acceleration was of greater relative importance for early acceleration performance than average resultant GRF magnitude during early acceleration, particularly from block starts (linear regression standardised $\beta$ coefficient for average $R F=0.82$, for resultant GRF magnitude $=0.10$ ). Direct measures of $D_{R F}$ and $R_{0}$ obtained from the block exit/initial push off and first four steps combined to explain 93-95\% of the variance in early acceleration performance, with $\mathrm{RF}_{0}$ being of greater relative importance for performance than $D_{R F}$. There was a near perfect correlation ( $r=0.94$ to 0.95$)$ between the macroscopic modelled $\mathrm{RF}_{\mathrm{MAX}}$ value $(\mathrm{RF}$ at $0.3 \mathrm{~s}$ based on a linear fit to modelled $\mathrm{RF}-\mathrm{v}_{\mathrm{H}}$ from $0.3 \mathrm{~s}$ onwards) and early acceleration performance. Whilst the ability to achieve high levels of resultant GRF magnitude cannot be ignored, particularly for standing starts, athletes should be encouraged to achieve a high initial ratio of forces during early acceleration before then focussing on arresting the decline in RF as the early acceleration phase progresses. Practitioners can use $\mathrm{RF}_{\mathrm{MAX}}$ from a simple macroscopic model fitted to the entire acceleration phase in field-based testing to provide a highly appropriate assessment of early acceleration performance. 


\section{Introduction}

Rapid acceleration from stationary is a fundamental ability for track sprinters and many team sport athletes. The techniques used during the early part of the acceleration phase are therefore often closely scrutinised in attempts to improve performance. By analysing external force data, it has been established that an athlete's "technical" ability to direct the ground reaction forces (GRFs) more horizontally (i.e. a higher ratio of horizontal-to-resultant forces (RF); Morin et al., 2011) is an important feature of their performance over the entire acceleration phase (Morin et al., 2011; Rabita et al., 2015). In quantifying this ability, a linear trendline is typically fitted to RF with respect to horizontal velocity $\left(\mathrm{v}_{\mathrm{H}}\right)$. The gradient of this trendline is then extracted as an indicator of force application technique throughout the acceleration phase and termed the rate of decline in $\mathrm{RF}\left(\mathrm{D}_{\mathrm{RF}}\right.$; Morin et al., 2011). Other measures which relate to the relative location of this relationship within the $R F-v_{H}$ space have also been extracted, including the theoretical maximal RF at null velocity from the linear RF$\mathrm{V}_{\mathrm{H}}$ fit $\left(\mathrm{RF}_{0}\right.$; Rabita et al., 2015), the maximal measured $\mathrm{RF}$ (Morin et al., 2011) or the mean $\mathrm{RF}$

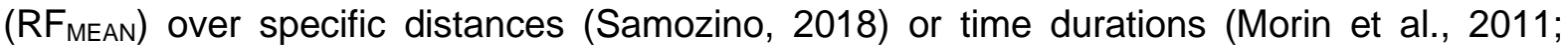
Bayne, 2018). Whilst these measures of maximal or mean RF ability may be more specific to early acceleration, they are typically determined from the linear function fitted to the RF- $\mathrm{v}_{\mathrm{H}}$ data over the entire acceleration phase, and thus RF values from later in the acceleration phase will influence them. It therefore remains unknown whether these measures provide the most appropriate representation of RF abilities during early acceleration, or whether they may be biased by abilities later in the acceleration phase (Samozino, 2018) and a specific fit to the early acceleration data could provide a more appropriate representation.

A simple macroscopic model based on a mono-exponential fit to the $\mathrm{v}_{\mathrm{H}}$-t profile (Samozino et al., 2016) has been widely used to obtain measures of $D_{R F}$ and $R F_{M A X}$ (the $R F$ value at $0.3 \mathrm{~s}$ based on a linear fit to modelled RF- $\mathrm{v}_{\mathrm{H}}$ from $0.3 \mathrm{~s}$ onwards), as well as some of the RF values reported above (Bayne, 2018; Samozino, 2018). These measures align well with values obtained directly from external force data (Samozino et al., 2016; Morin et al., 2019). However, 
they have been the subject of some discussion because of the simplicity of the underlying $\mathrm{V}_{\mathrm{H}^{-}}$ t model (Haugen et al., 2019a), the estimation of $\mathrm{RF}_{\mathrm{MAx}}$ at low speeds where the modelled RF- $\mathrm{v}_{\mathrm{H}}$ relationship deviates slightly from being linear, and the lack of consideration of the effects of within-step kinetics (Pavei et al., 2019). This simple macroscopic modelling approach is also subject to the potential aforementioned issues regarding the influence of data from all later parts of the acceleration phase if interested solely in early acceleration.

The methods used to determine a single RF value for each step from direct measurements of GRF have not been consistent between studies. For example, Rabita et al. (2015) calculated instantaneous RF and took the mean value over each stance phase as step-averaged RF, Morin et al. $(2011 ; 2012)$ used the mean horizontal and resultant forces over each stance phase to determine step-averaged $\mathrm{RF}^{1}$, and Samozino et al. (2016) and Morin et al. (2019) used the mean horizontal and vertical forces over each step. Whilst step-averaged RF values will not differ if average forces are determined over just the stance phase or over an entire step (i.e. stance + aerial phases), the inclusion of the aerial phase will influence calculations which are based on the averaging of instantaneous RF data because a value would have to be assigned to RF during the aerial phase and incorporated in to this average. This explains why studies which have used average instantaneous RF data (e.g. Rabita et al., 2015) have done so over just the stance phase. Furthermore, using the mean of the vertical forces and the mean of the horizontal forces to determine a mean resultant force (e.g. Samozino et al., 2016; Morin et al., 2019) will typically yield a different denominator for determining stepaveraged RF compared with using the mean of the instantaneous resultant forces (e.g. Morin et al., 2011; 2012). As the approaches used to determine step-averaged RF are all computationally different, they may yield a different value for step-averaged RF, and this could consequently also lead to different applied interpretations of an athlete's "technical" ability.

\footnotetext{
${ }^{1}$ Morin et al. (2011) also presented a figure of the instantaneous RF trace throughout an entire contact phase but these instantaneous RF data were for illustrative purposes and were not used in the calculation of step-averaged RF.
} 
The overriding aim of this study was to establish the importance of the direction of force application to sprint performance during early acceleration ( $\mathrm{H} 2$ and $\mathrm{H} 3)$, defined as the initial push-off/block exit and the first four steps. In order to achieve this, it was first necessary to assess the effects of different input data and processing calculations on the obtained RF metrics $(\mathrm{H} 1)$ :

H1) The RF- $v_{H}$ profile will differ when step-averaged RF is calculated from instantaneous RF data compared with when calculated from step-averaged force data.

H2) Average RF will be of greater importance for early acceleration performance than average absolute GRF magnitude.

H3) $R F_{0}$ and $D_{R F}$ determined from early acceleration data will be of comparable importance in achieving high early acceleration performance.

Each of the above hypotheses were addressed using data for both standing and block starts (separately) in order to widen the generalisability of the obtained understanding. Finally, the

$D_{\mathrm{RF}}$ and $\mathrm{RF}_{\mathrm{MAX}}$ outputs obtained from a simple macroscopic model fitted to the $\mathrm{v}_{\mathrm{H}}$-time data over the entire acceleration phase (Samozino et al., 2016) were compared against the force plate determined RF and performance variables during early acceleration to assess how well commonly collected field-based data corresponded to directly measured GRF data from just the early acceleration phase.

\section{Methods}

Participants

Twenty-four male sprinters (mean \pm SD: age $=20 \pm 1$ years; height $=1.73 \pm 0.06 \mathrm{~m}$; mass = $65.7 \pm 4.0 \mathrm{~kg} ; 100 \mathrm{~m}$ personal best $=11.26 \pm 0.39 \mathrm{~s})$ provided informed consent to participate in this study. All sprinters wore their own spiked shoes and were experienced with standing and block starts. All procedures were approved by the research ethics committee of the National Institute of Fitness and Sports in Kanoya. 


\section{Protocol}

All sprinters completed a self-directed warm-up before performing four maximal effort $60 \mathrm{~m}$ sprints on an indoor track over a $52 \mathrm{~m}$ series of force platforms (TF-3055, TF-32120, TF90100, Tec Gihan, Uji, Japan). Two sprints were from a block start (preferred individual block set-up) and two were from a two-point standing start in a relatively crouched posture. The order of conditions was randomised and rest of at least 10 minutes was provided between sprints. An electric starting gun was used to synchronously initiate the GRF data collection $(1000 \mathrm{~Hz})$ and emit an auditory starting signal, and data collection was manually terminated once the sprinter had passed $60 \mathrm{~m}$. All raw GRF data were stored and exported for subsequent analysis in Matlab $^{\circledR}\left(\right.$ R2019b, MathWorks ${ }^{\circledR}$, Natick, MA).

\section{Data analysis}

Based upon an initial visual inspection of the GRF data, 12 standing start trials were removed because the sprinter was clearly not stationary immediately after the starting signal. Six of these comprised both trials for three sprinters and thus $n=21$ for all standing start analyses. For the sprinters who the other six trials corresponded to, their successful trial was used as the sole input for all standing start analyses. Any systematic offsets (all $<5 \mathrm{~N}$ ) were removed from the respective channels of the raw GRF data. For all block starts, movement onset was determined as the first sample of the first $\geq 0.1 \mathrm{~s}$ period where the raw antero-posterior GRF consistently exceeded two standard deviations above the mean raw antero-posterior force during the first $0.1 \mathrm{~s}$ of the trial. The same process was initially applied to the standing start data but owing to considerable variation in the standing start GRF profiles between sprinters, it was decided to manually visually identify movement onset as the start of the clear rapid increase in anterior GRF (see Reflections, limitations and future directions for further consideration of this issue).

The vertical and antero-posterior GRF data from the start signal to the end of data capture were filtered using a $4^{\text {th }}$ order low-pass Butterworth filter with a cut-off frequency of $70 \mathrm{~Hz}$. 
Each toe-off (or block exit for the first event of the block start trials) and touchdown event was identified using a $25 \mathrm{~N}$ threshold in the filtered vertical GRF data. Horizontal velocity was then determined from the filtered antero-posterior GRFs using the impulse-momentum relationship accounting for the influence of air resistance (Colyer et al., 2018). Drag force was estimated using the equations described by Samozino et al. (2016) with directly measured ambient temperature and atmospheric pressure used as inputs for each participant. The resulting 'instantaneous' (i.e. $1000 \mathrm{~Hz}$ ) $\mathrm{V}_{\mathrm{H}}$-t profile for each trial was visually compared against the $\mathrm{v}_{\mathrm{H}} \mathrm{t}$ profile from average step velocities and times obtained from touchdown and toe-off events and the antero-posterior centre of pressure location. This served as a visual quality check of the instantaneous $\mathrm{v}_{\mathrm{H}}$-t profile to ensure it was not markedly affected by inaccurate mass values or movement prior to the start of recording.

Step-averaged $\mathrm{v}_{\mathrm{H}}$ and RF were then determined for each step in every trial, starting with the block exit (for block start trials) or initial push-off (for standing start trials). In order to address the first hypothesis, step-averaged RF was determined using two different approaches. In the first approach, instantaneous RF was determined from the filtered $1000 \mathrm{~Hz}$ antero-posterior and vertical GRF data, and the mean instantaneous RF was determined during each stance phase to obtain step-averaged RF from instantaneous RF data (stance phase data only were used in this calculation for reasons outlined in the introduction). In the second approach, stepaveraged antero-posterior force was divided by the resultant of the step-averaged anteroposterior and vertical forces, consistent with the procedures of Samozino et al. (2016) and Morin et al. (2019), to obtain step-averaged RF from step-averaged force data. Because stepaveraged RF was obtained from just the stance phase in the first approach described above, step-averaged $v_{H}$ for this first approach was obtained from the average instantaneous $v_{H}$ during just the stance phase, whereas for the second approach which used step-averaged force data it was determined from the entire step (i.e. from one touchdown to the next). This ensured that the RF and $V_{H}$ values used in the determination of each $R F-v_{H}$ relationship corresponded directly to one another. The step with the highest velocity was identified and a 
linear fit to the RF- $v_{H}$ data from the initial block exit/push-off step to this step was performed separately for the RF- $\mathrm{V}_{\mathrm{H}}$ data from each of the two approaches for each trial. The gradient was extracted as a modelled measure of the rate of decline in RF over the entire acceleration phase (DRF-FP_all $)$ and the $y$-intercept was extracted as a measure of theoretical maximal RF based on data from the entire acceleration phase $\left(\mathrm{RF}_{0-\mathrm{FP} \_ \text {all }}\right)$. To compare the $\mathrm{RF}-\mathrm{V}_{\mathrm{H}}$ profiles between the two calculation approaches and enable the first hypothesis to be addressed, $D_{\mathrm{RF}}$ FP_all and $\mathrm{RF}_{0-\mathrm{FP} \_ \text {all }}$ were compared between the two calculation methods using a pairedsamples t-test, and the systematic and random differences (95\% limits of agreement) were calculated using a Bland-Altman analysis.

To address the second and third hypotheses which related specifically to the importance of RF during early acceleration, a direct measure of early acceleration performance was required. The velocity of the sprinter at the $4^{\text {th }}$ step toe-off and the time taken to reach this toeoff (relative to the instant of movement onset) were extracted and used to determine the normalised average horizontal external power (NAHEP) produced by the sprinter over the entire period from movement onset to $4^{\text {th }}$ step toe-off, following the procedures outlined by Bezodis et al. (2010) and estimating leg length as 53\% of stature (Winter, 2005). The stepaveraged RF values (using the step-averaged force data - see Discussion for justification and Appendix (Table A1) for selected results based on alternative outputs using step-averaged RF values from instantaneous $R F$ data) from the same early acceleration phase (i.e. block exit/push-off step and the first four steps on the track) were then extracted. Mean RF (RFMEANFP) during early acceleration was calculated from the average RF values during the block exit/push-off step and each of the first four steps on the track. Mean absolute force magnitude (FTOT-FP) was calculated from the average resultant force produced during each of these same five phases. These were then entered in to a stepwise multiple linear regression (SPSS ${ }^{\circledR} \mathrm{V}$. 26, IBM ${ }^{\circledR}$, Armonk, NY) to determine the relative importance of force magnitude $\left(\mathrm{F}_{\text {TOT-FP }}\right)$ and direction of force application (RFMEAN-FP), both calculated from directly measured GRF data, to performance (NAHEP) during just the early acceleration phase in order to address the second 
hypothesis. A linear trendline was then fitted through the five $R F$ and $v_{H}$ values (these calculations were also performed without the datapoints from the block exit/push-off step but as the relationships with performance were typically weaker - see Appendix (Table A1) - only the results using the trendlines fitted through all five values were retained for presentation in this manuscript). The gradient $\left(\mathrm{D}_{\mathrm{RF}-\mathrm{FP}}\right)$ and $\mathrm{y}$-intercept $\left(\mathrm{RF}_{0-\mathrm{FP}}\right)$ were extracted as measures of RF ability during early acceleration, and these were entered into a stepwise multiple linear regression to determine their share of the explained variance in performance (NAHEP) during the early acceleration phase to address the third hypothesis.

Finally, in order to assess how well the RF outputs obtained from a simple macroscopic model which was fitted to the $\mathrm{V}_{\mathrm{H}}$-time data over the entire acceleration phase (Samozino et al., 2016) corresponded to those directly obtained from the force platform data during just early acceleration, the force platform determined $\mathrm{v}_{\mathrm{H}}$-time data from movement onset to toe-off of the step with the highest stance-averaged velocity were fitted with a mono-exponential function (Samozino et al., 2016). The modelled horizontal force-time data were determined based on these fitted velocity data and the modelled effects of drag, with step-averaged vertical force assumed to be equal to body weight throughout (Samozino et al., 2016). The instantaneous modelled RF time-history throughout the sprint was then determined (Morin et al., 2011). The value of this $R F$ function at $0.3 \mathrm{~s}$ was extracted $\left(R F_{M A X-M}\right)$ as a representation of the RF value during the block exit/push-off step (Samozino et al., 2016). A linear fit to the modelled RF- $\mathrm{v}_{\mathrm{H}}$ data (ignoring the first $0.3 \mathrm{~s}$ of data after movement onset) was performed and the gradient of this function was extracted $\left(D_{R F-M}\right)$. Bivariate correlations between the force plate determined $R F$ variables during early acceleration $\left(\mathrm{RF}_{\mathrm{MEAN}-\mathrm{FP}}, \mathrm{RF}_{0-\mathrm{FP}}, \mathrm{D}_{\mathrm{RF}-\mathrm{FP}}\right)$ and the simple macroscopic model variables over the whole acceleration phase (RF $\left.F_{M A X-M}, D_{R F-M}\right)$ were performed, as were correlations between the simple macroscopic model variables and early acceleration NAHEP. 
For all analyses undertaken, mean values of the two trials for each sprinter in each condition were used (aside from six sprinters' standing starts where only one trial was available for reasons described previously). Statistical significance was accepted at $p<0.05$, and thresholds for correlation coefficients (Pearson's $r)$ were defined as trivial $(r<0.1)$, small $(0.1$ $\leq r<0.3)$, moderate $(0.3 \leq r<0.5)$, large $(0.5 \leq r<0.7)$, very large $(0.7 \leq r<0.9)$, nearly perfect $(0.9 \leq r<1.0)$ or perfect $(r=1.0$; Hopkins, 2014).

\section{Results}

The normalised average external power production over the initial push off and first four steps was similar between the block and standing starts (Table 1). When expressed relative to time of movement onset and centre of mass (CM) position at this instant, block starts led to a shorter time to the end of the fourth contact, but less horizontal CM displacement and a lower $\mathrm{CM}$ velocity at the end of this contact (Table 1).

There were very large relationships $(r=0.75$ to 0.88 , all $p<0.001)$ for both the $R_{0-F P \_a l l}$ and $D_{\text {RF-FP_all }}$ measures when compared between the two calculation methods used to obtain stepaveraged $\mathrm{RF}^{2}$. However, there was a significant (all $\left.p<0.001\right)$ difference between the two methods for both measures (Table 1). For all trials, the method which used the instantaneous RF data demonstrated a lower $\mathrm{RF}_{0-\mathrm{FP} \_ \text {all }}$ and a shallower $\mathrm{D}_{\mathrm{RF}-\mathrm{FP} \_a l l}$ than the method which used the step-averaged force data (Table 1; Figures 1 and 2). When compared between the two calculation methods for obtaining step-averaged RF, the systematic difference $\pm 95 \%$ limits of agreement for $\mathrm{D}_{\mathrm{RF}-\mathrm{FP} \_a l l}$ was $-1.7 \pm 0.4 \% \cdot \mathrm{s} / \mathrm{m}$ (blocks) and $-1.7 \pm 0.6 \% \cdot \mathrm{s} / \mathrm{m}$ (standing), and for $\mathrm{RF}_{0-\mathrm{FP} \_a l l}$ was $9.2 \pm 3.3 \%$ (blocks) and $8.6 \pm 4.3 \%$ (standing; Figure 1 ).

\footnotetext{
2 These $r$ values reported here correspond to the entire acceleration phase including the initial pushoff/block exit. Pearson's correlations between the outputs from the two calculation methods are presented in the Appendix (Table A2) for four different combinations of input data (i.e. from the whole acceleration phase or from the early acceleration phase only, both inclusive and exclusive of the initial push-off/block exit).
} 
Table 1. Summary of initial acceleration performance measures and ratio of forces measures (all mean \pm SD) determined directly from the force platform data (over both the entire acceleration phase and the initial acceleration phase) and from the simple macroscopic model.

\begin{tabular}{|c|c|c|}
\hline & Block starts & Standing starts \\
\hline NAHEP up to end of $4^{\text {th }}$ contact & $0.57 \pm 0.04$ & $0.57 \pm 0.05$ \\
\hline Velocity at end of $4^{\text {th }}$ contact $(\mathrm{m} / \mathrm{s})$ & $6.53 \pm 0.20$ & $6.75 \pm 0.19$ \\
\hline Time to end of $4^{\text {th }}$ contact $^{\wedge}(\mathrm{s})$ & $1.28 \pm 0.07$ & $1.37 \pm 0.08$ \\
\hline Displacement at end of $4^{\text {th }} \operatorname{contact}^{\wedge}(\mathrm{m})$ & $5.08 \pm 0.35$ & $5.62 \pm 0.32$ \\
\hline $\begin{array}{l}D_{\text {RF-FP_all }} \text { using step-averaged RF from } \\
\text { instantaneous RF data }(\% \cdot s / m)\end{array}$ & $-5.6 \pm 0.5$ & $-5.4 \pm 0.04$ \\
\hline $\begin{array}{l}D_{\text {RF-FP_all using step-averaged RF from }} \\
\text { step-averaged force data }(\% \cdot s / m)\end{array}$ & $-7.4 \pm 0.5^{\star \star *}$ & $-7.1 \pm 0.4^{* \star *}$ \\
\hline $\begin{array}{l}\mathrm{RF}_{0-\mathrm{FP} \text { _all }} \text { using step-averaged RF from } \\
\text { instantaneous } \mathrm{RF} \text { data (\%) }\end{array}$ & $64.7 \pm 2.7$ & $63.8 \pm 3.6$ \\
\hline $\begin{array}{l}\mathrm{RF}_{0-\mathrm{FP}} \text { all } \text { using step-averaged RF from } \\
\text { step-averaged force data (\%) }\end{array}$ & $73.9 \pm 3.2^{\star \star \star}$ & $72.4 \pm 3.6^{\star * *}$ \\
\hline $\mathrm{RF}_{\mathrm{MEAN}-\mathrm{FP}^{\dagger}(\%)}$ & $40.2 \pm 1.4$ & $37.9 \pm 1.2$ \\
\hline $\mathrm{F}_{\text {TOT-FP }}(\mathrm{BW})$ & $1.11 \pm 0.02$ & $1.10 \pm 0.02$ \\
\hline$D_{\mathrm{RF}-F P^{\dagger}}(\% \cdot \mathrm{s} / \mathrm{m})$ & $-7.9 \pm 0.7$ & $-7.0 \pm 0.9$ \\
\hline $\mathrm{RF}_{0-\mathrm{FP}}^{\dagger}(\%)$ & $76.0 \pm 3.6$ & $71.8 \pm 5.3$ \\
\hline $\mathrm{D}_{\mathrm{RF}-\mathrm{M}}(\% \cdot \mathrm{s} / \mathrm{m})$ & $-7.6 \pm 0.5$ & $-7.6 \pm 0.6$ \\
\hline $\mathrm{RF}_{\text {MAX-M }}(\%)$ & $55.0 \pm 1.6$ & $55.1 \pm 2.5$ \\
\hline
\end{tabular}

$\wedge$ Relative to movement onset.

Asterisks denote significant difference from corresponding value calculated using step-averaged RF from instantaneous RF data based on results of a paired-samples t-test $\left({ }^{*} p<0.05 ;{ }^{* *} p<0.01 ;{ }^{* * *} p<0.001\right)$.

${ }^{\dagger}$ determined using the step-averaged ratio of forces from step-averaged force data.

NAHEP: Normalised average horizontal external power.

$\mathrm{D}_{\mathrm{RF}-\mathrm{FP} \text { all }}$ : the rate of decline in ratio of forces over the entire acceleration phase, determined as the gradient of the linear fit to the ratio of forces-horizontal velocity data;

$\mathrm{RF}$ : ratio of forces;

$\mathrm{RF}_{0 \text {-FP_all: }}$ theoretical maximal ratio of forces based on data from the entire acceleration phase, determined as the $y$-intercept of the linear fit to the ratio of forces-horizontal velocity data.

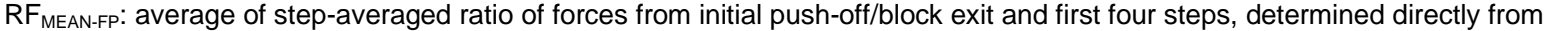
force platform data;

$\mathrm{F}_{\mathrm{TOT}-\mathrm{FP}}$ : average of step-average resultant force from initial push-off/block exit and first four steps, determined directly from force platform data;

BW: body weight;

$\mathrm{D}_{\mathrm{RF}-\mathrm{FP}}$ : the rate of decline in ratio of forces, determined as the gradient of the linear fit to the ratio of forces-horizontal velocity data from initial push-off/block exit and first four steps;

$\mathrm{RF}_{0 \text {-FP: }}$ theoretical maximum ratio of forces at zero velocity, determined as the $y$-intercept of the linear fit to the ratio of forceshorizontal velocity data from initial push-off/block exit and first four steps.

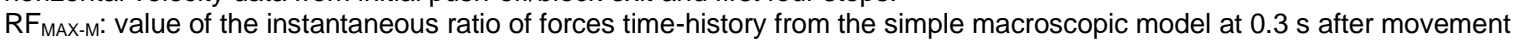
onset;

$\mathrm{D}_{\mathrm{RF}-\mathrm{M}}$ : gradient of the linear fit to the ratio of forces-horizontal velocity data from the simple macroscopic model, excluding the first $0.3 \mathrm{~s}$ of data after movement onset in this fit. 
a)

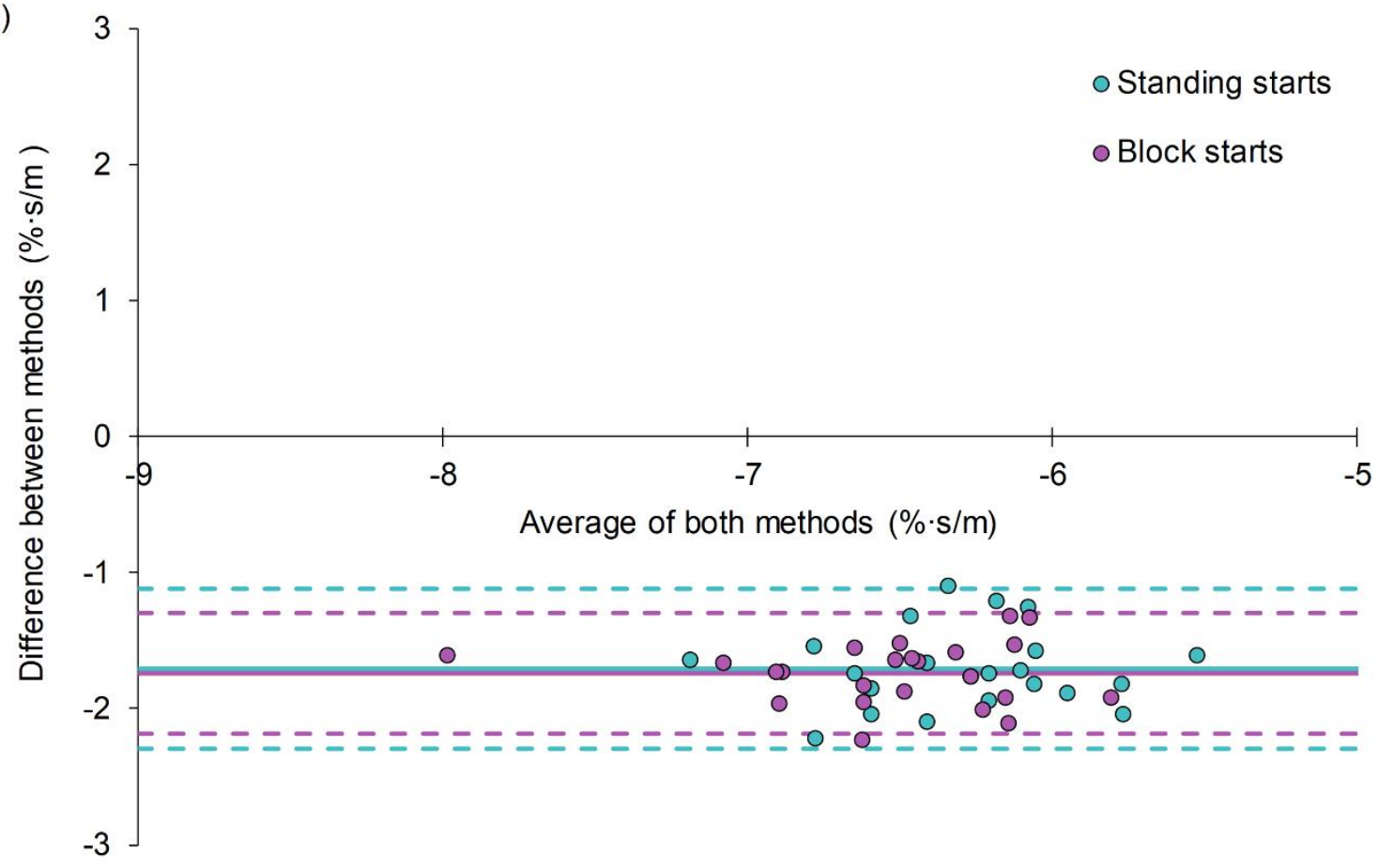

b)

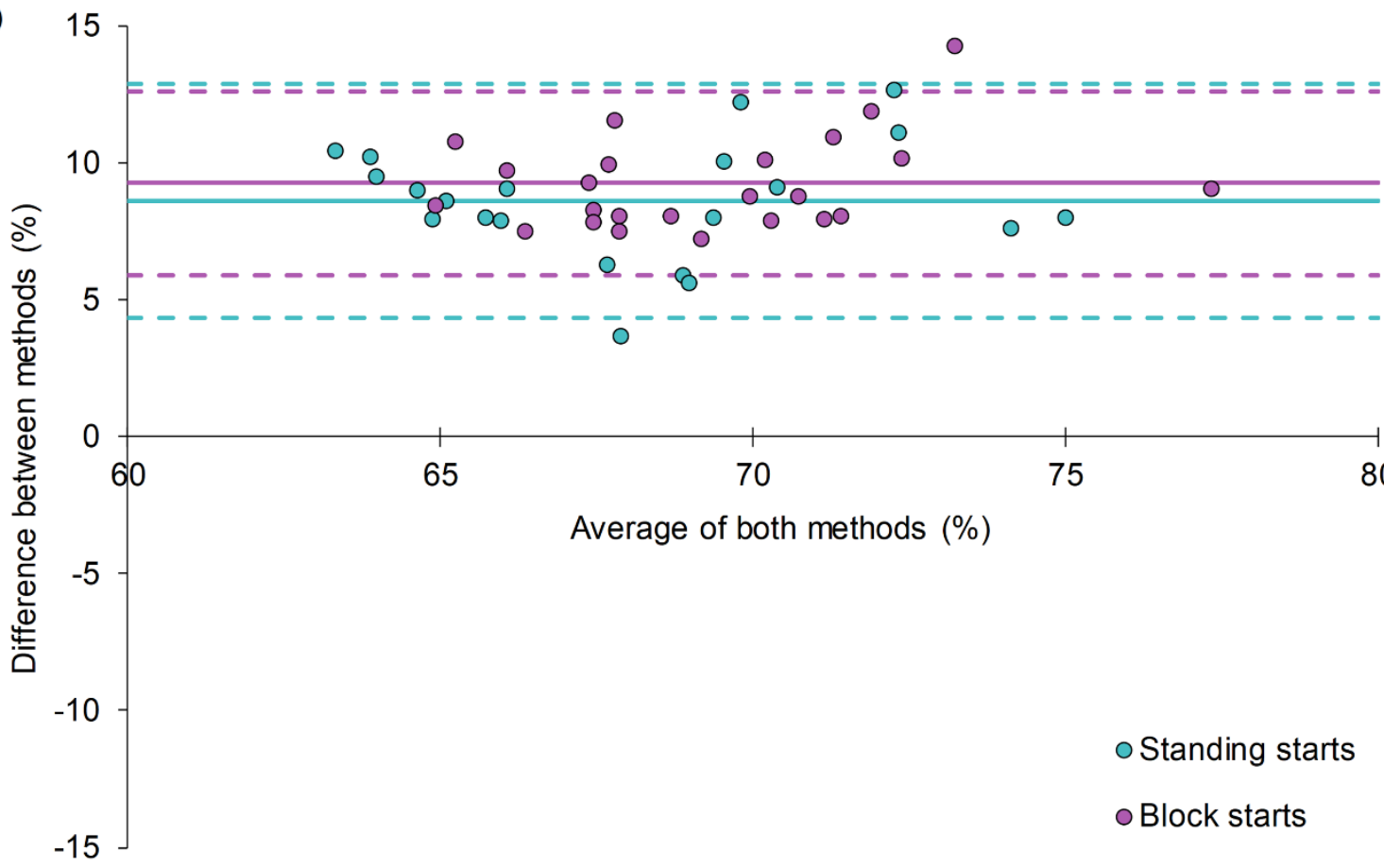

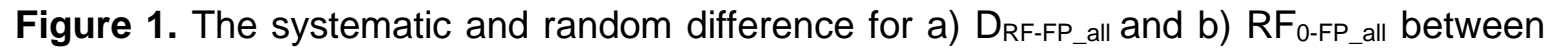
the two step-averaged RF calculation methods. Differences are determined as the values from the step-averaged RF calculated from step-averaged force data minus those from the step-averaged RF calculated from instantaneous RF data. The solid horizontal line represents the systematic bias and the dashed horizontal lines represent the $95 \%$ limits of agreement. 


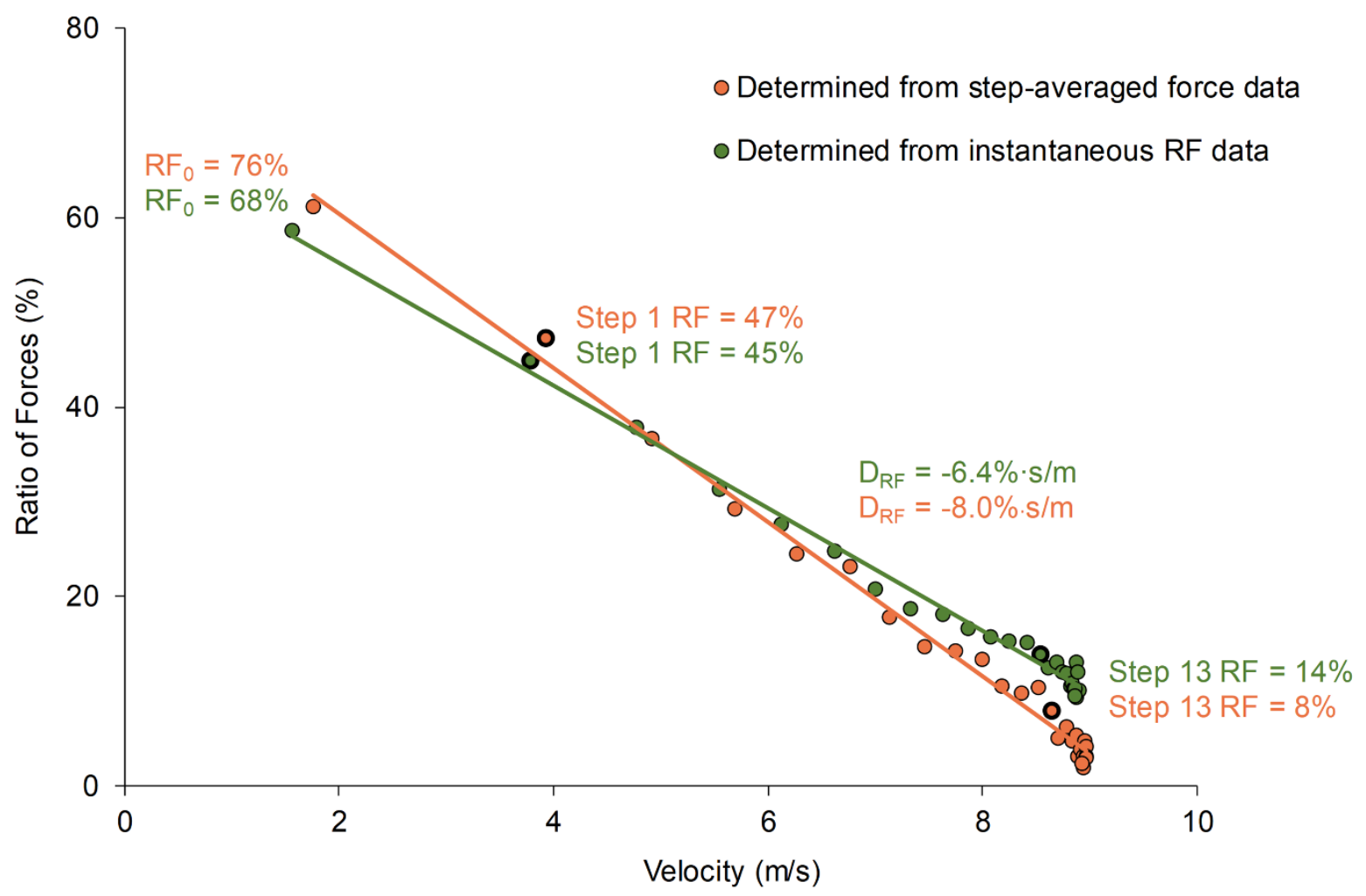

Figure 2. Ratio of forces-horizontal velocity relationships compared between the two ratio of forces calculation methods for a typical trial from blocks. The $R F_{0}$ and $D_{R F}$ values presented in this figure are based on data from the entire acceleration phase (i.e. $R F_{0-F P_{-} a l l}$ and $D_{\text {RF-FP_all }}$. Step 1 and Step 13 step-averaged RF values are also stated and correspond to the respective data points with a bold outline.

There was a very large correlation between mean step-averaged RF (using step-averaged force data) obtained from the force platforms during early acceleration (RF $F_{M E A N-F P}$ ) and performance (NAHEP) from both block and standing starts $(r=0.88$ and 0.84 , respectively, both $p<0.001)$. The relationships between $R F_{M E A N-F P}$ and the average resultant force magnitude ( $\left.\mathrm{F}_{\text {TOT-FP }}\right)$ were large for both the standing $(r=0.63, p<0.01)$ and block $(0.59, p<$ $0.01)$ starts. The addition of $F_{T O T-F P}$ to $R F_{M E A N-F P}$ in a multiple regression to predict performance (blocks: $F=35.5, p<0.001$; standing: $F=38.7, p<0.001$ ) only increased the explained variance for the standing start data, and only by $10 \%$ (to an adjusted $\mathrm{R}^{2}$ of $79 \%$ ). For the block starts, the standardised $\beta$-coefficient for $\operatorname{RF}_{\text {MEAN-FP }}(0.82, p<0.001)$ was over eight times greater than that for $\mathrm{F}_{\mathrm{TOT}-\mathrm{FP}}(0.10, \mathrm{p}=0.47)$, whereas for the standing starts the magnitudes of these coefficients were much closer $\left(0.58, p<0.001\right.$ for $R F_{\text {MEAN-FP }}$ and $0.42, p<0.01$ for F 
When investigating the relative importance of theoretical peak $R F\left(R F_{0-\mathrm{FP}}\right)$ and the rate of decline in $\mathrm{RF}\left(\mathrm{D}_{\mathrm{RF}-\mathrm{FP}}\right)$ for early acceleration performance, $\mathrm{RF}_{0-\mathrm{FP}}$ was more strongly correlated to NAHEP than $D_{\text {RF-FP }}$ (Table 2; Figures 3-4). The addition of $D_{\text {RF-FP }}$ to $R F_{0-F P}$ in a multiple regression to predict performance (Table 2) increased the explained variance from $32 \%$ to $93 \%$ for the block starts, and from $60 \%$ to $95 \%$ for the standing starts (Table 2; Figure 5; Figure S1). Based on the standardised $\beta$-coefficients, $R F_{0-F P}$ had greater importance in determining performance than D $\mathrm{RF-FP}$, but only by a factor of 1.3 to 1.4 (Table 2).

Table 2. Regression results between force plate determined RF measures and performance (NAHEP) during early acceleration.

Block starts

$0.59^{* *}$

$-0.04$

$11.7^{\star \star}$

$32 \%$ $161.8^{\star \star \star}$ $93 \%$ $95 \%$

Adjusted $\mathrm{R}^{2}\left(\mathrm{RF}_{0-\mathrm{FP}} \& \mathrm{D}_{\mathrm{RF}-\mathrm{FP}}\right)$

$\mathrm{RF}_{0 \text {-FP }} \beta$-coefficient $1.69^{\star \star *}$ $2.40^{\star * *}$

$D_{\text {RF-FP }} \beta$-coefficient
Standing starts

$0.79^{\star \star \star}$

$-0.55^{\star *}$
Adjusted $\mathrm{R}^{2}$ ( $\mathrm{RF}_{0 \text {-FP }}$ only) $60 \%$

$\mathrm{F}\left(\mathrm{RF}_{0-\mathrm{FP}} \& \mathrm{D}_{\mathrm{RF}-\mathrm{FP}}\right)$ 


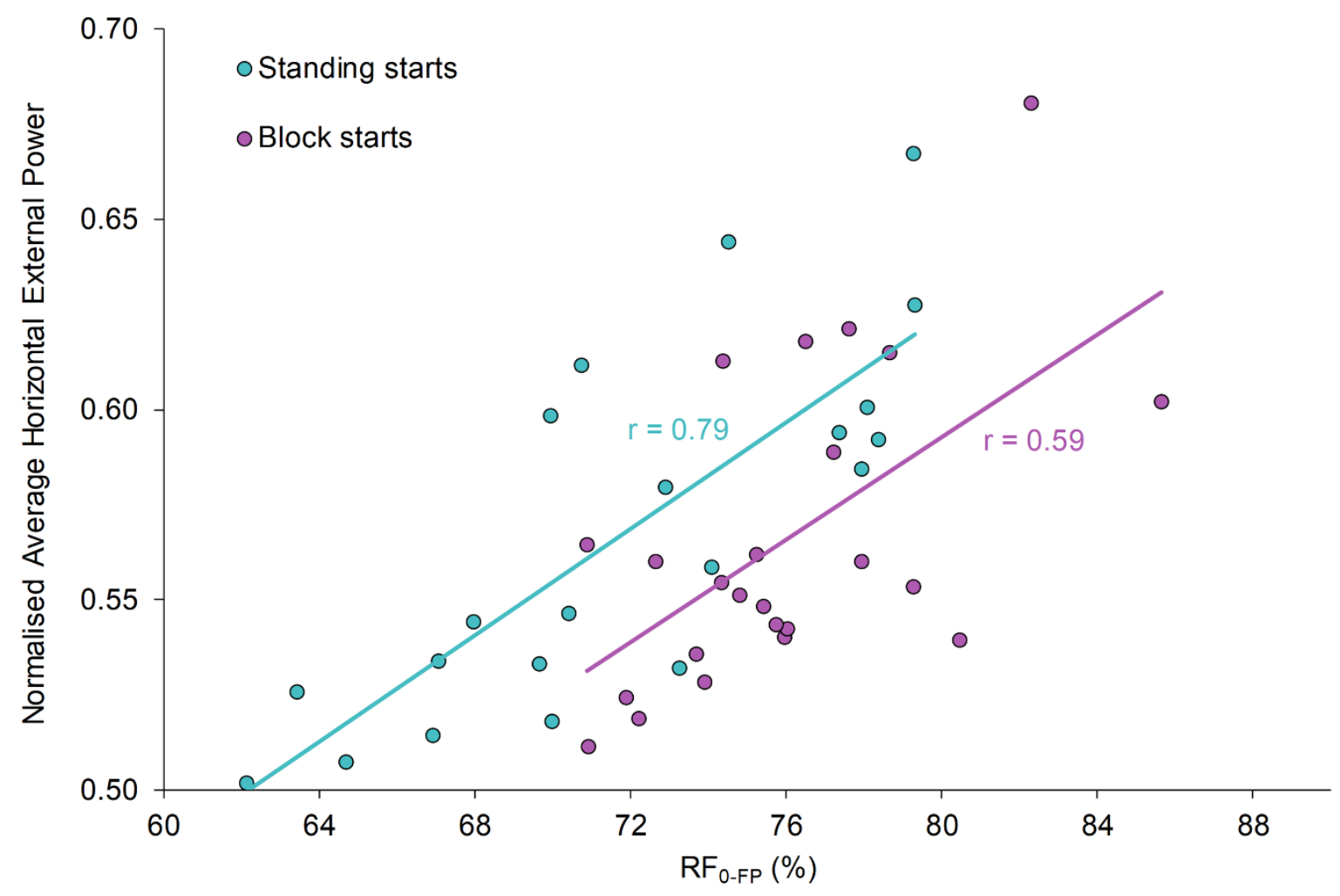

Figure 3. $\mathrm{RF}_{0-\mathrm{FP}}$ from the slope fitted to $\mathrm{RF}-\mathrm{v}_{\mathrm{H}}$ data over the initial push-off/block exit and first four steps versus performance (NAHEP) up to the end of the $4^{\text {th }}$ step.

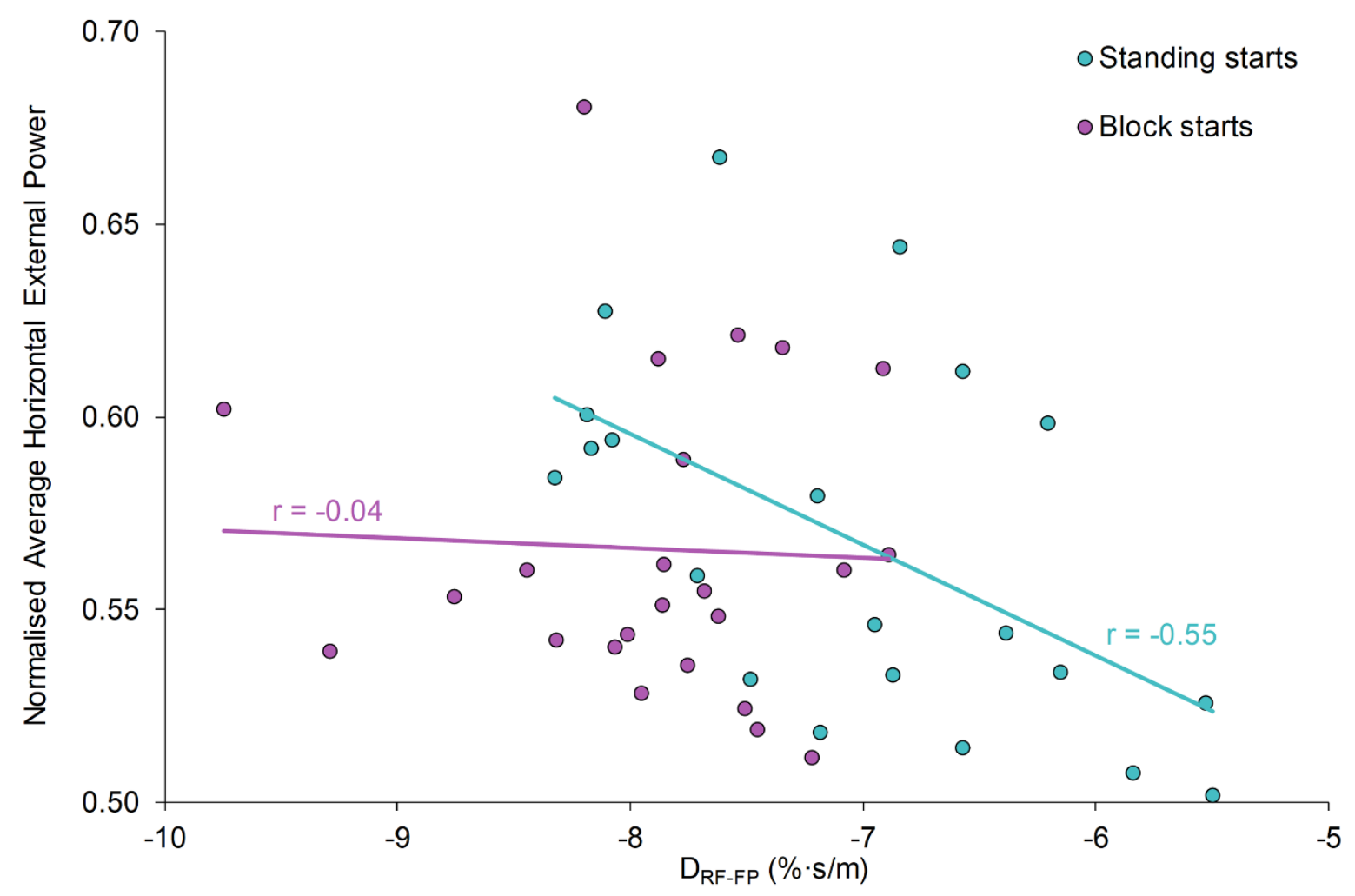

Figure 4. $D_{R F-F P}$ from the slope fitted to RF- $V_{H}$ data over the initial push-off/block exit and first four steps versus performance (NAHEP) up to the end of the $4^{\text {th }}$ step. 
a)

- Standing starts

- Block starts

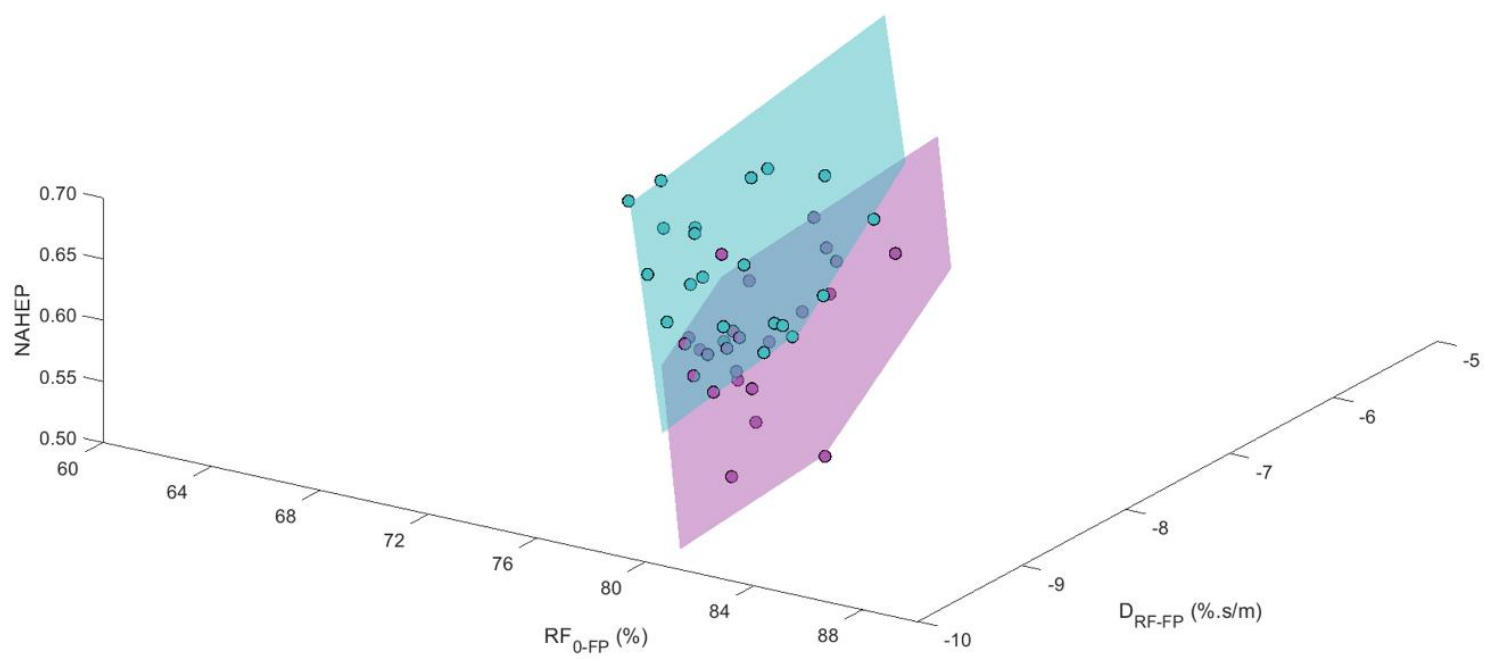

b)

- Standing starts

- Block starts

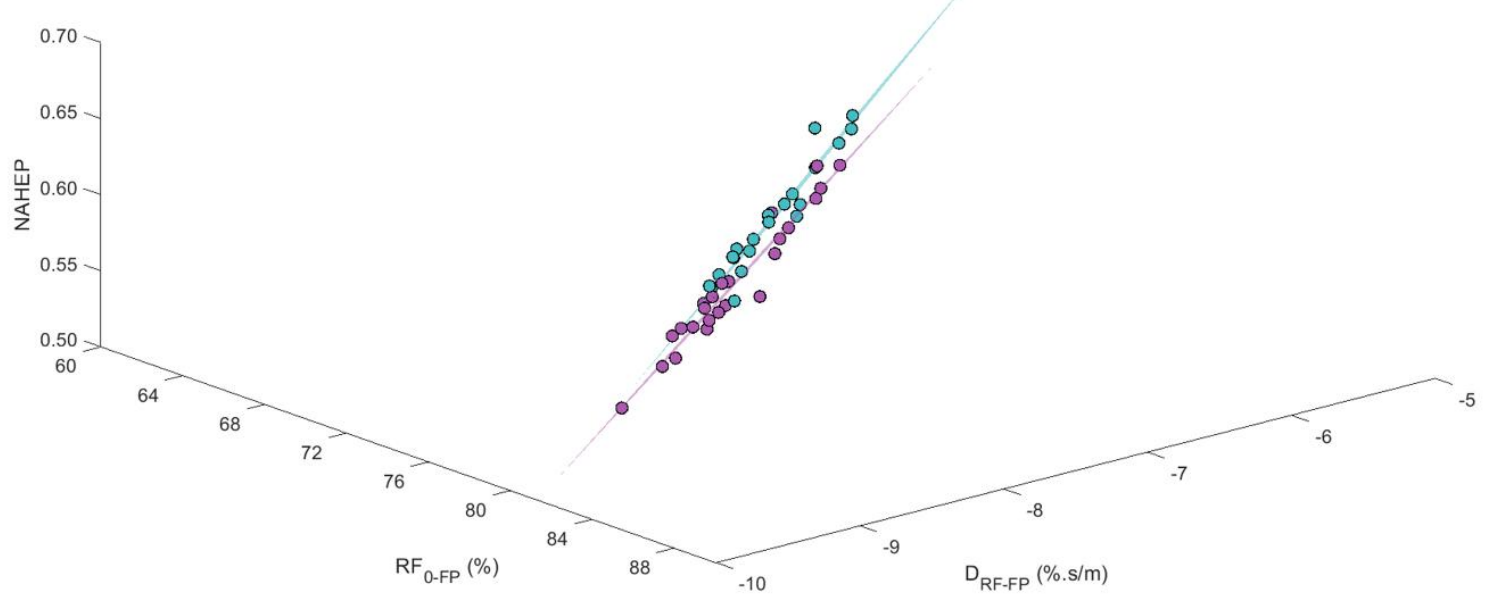

Figure 5. The combination of $D_{R F-F P}$ and $R_{0-F P}$ from the slope fitted to $R F-v_{H}$ data over the initial push-off/block exit and first four steps versus performance (NAHEP) up to the end of the $4^{\text {th }}$ step.

Note: $a$ and $b$ are two different views of the same 3D plot with the best-fit orthogonal plane fitted to the data. Data also correspond directly to those in the 2D views in Figures 3 and 4. See video of Figure 5 in supplementary files (Figure S1) for further views of these data. 
When analysing the simple macroscopic model outputs $\left(\mathrm{RF}_{\mathrm{MAX}-\mathrm{M}}, \mathrm{D}_{\mathrm{RF}-\mathrm{M}}\right)$ determined from the step averaged $\mathrm{RF}$ and $\mathrm{V}_{\mathrm{H}}$ data across the whole acceleration phase, there was a nearly perfect relationship between $\mathrm{RF}_{\mathrm{MAX}-\mathrm{M}}$ and early acceleration performance from block and standing starts $\left(r=0.94\right.$ and 0.95 , respectively, both $p<0.001$; Table 3). When RF $F_{\text {MAX-M }}$ was combined with $\mathrm{D}_{\mathrm{RF}-\mathrm{M}}$ to predict NAHEP, the explained variance increased slightly to $92-95 \%$, but $\mathrm{RF}_{\mathrm{MAX}}$ м clearly had the greater importance in determining performance based on the standardised $\beta$-coefficients which were greater by a factor of 3.6 for both block and standing starts (Table 3).

Table 3. Regression results between simple macroscopic model determined RF measures and performance (NAHEP) during early acceleration.

\begin{tabular}{|c|c|c|}
\hline & Block starts & Standing starts \\
\hline$r$ for $R_{\text {MAX-M }}$ vs. NAHEP & $0.94^{* * *}$ & $0.95^{* * *}$ \\
\hline$r$ for $D_{\text {RF-M }}$ vs. NAHEP & $-0.42^{*}$ & $-0.75^{\star * *}$ \\
\hline$F\left(R_{M A X-M} \& D_{R F-M}\right)$ & $206.9^{\star \star \star}$ & $117.0^{\star \star \star}$ \\
\hline Adjusted $\mathrm{R}^{2}$ & $95 \%$ & $92 \%$ \\
\hline $\mathrm{RF}_{\text {MAX-M }} \beta$-coefficient & $1.15^{\star \star \star}$ & $1.24^{\star * *}$ \\
\hline$D_{\text {RF-M }} \beta$-coefficient & $0.32^{* * *}$ & $0.34^{*}$ \\
\hline
\end{tabular}

The simple macroscopic model outputs (from the entire acceleration phase) were then correlated with the force platform outputs (from the early acceleration phase). The relationships between comparable parameters were typically very large but there were no near perfect correlations between RF properties during early acceleration and those obtained over the entire acceleration phase from a simple macroscopic model (Table 4) .

\footnotetext{
${ }^{3}$ Pearson's correlations ( $r$ ) using data obtained from the force platforms over the entire acceleration phase versus the simple macroscopic model are included in the Appendix (Table A3).
} 
Table 4. Pearson's correlations ( $r$ ) between force plate determined and simple macroscopic model RF variables for block and standing starts.

\begin{tabular}{|c|c|c|c|c|c|c|c|}
\hline \multirow{2}{*}{\multicolumn{2}{|c|}{ Blocks }} & \multicolumn{2}{|c|}{ Simple macroscopic model } & \multirow{2}{*}{\multicolumn{2}{|c|}{ Standing }} & \multicolumn{2}{|c|}{ Simple macroscopic model } \\
\hline & & RF & DRF-M & & & RFMAX-M & DRF-M \\
\hline \multirow{3}{*}{ 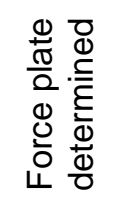 } & $\mathrm{RF}_{\mathrm{MEAN}-\mathrm{FP}}$ & $0.77^{\star \star \star}$ & -0.23 & \multirow{3}{*}{ 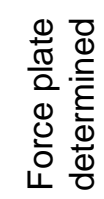 } & RF $_{\text {MEAN-FP }}$ & $0.75^{\star \star \star}$ & $-0.58^{\star *}$ \\
\hline & $\mathrm{RF}_{0-\mathrm{FP}}$ & $0.70^{\star \star \star}$ & $-0.59^{* *}$ & & $\mathrm{RF}_{0-\mathrm{FP}}$ & $0.89^{\star \star \star}$ & $-0.84^{\star \star *}$ \\
\hline & $D_{\text {RF-FP }}$ & -0.20 & $0.46^{*}$ & & DRF-FP & $-0.70^{* * *}$ & $0.73^{\star \star \star}$ \\
\hline
\end{tabular}

\section{Discussion}

This study addressed a series of hypotheses which initially related to the choice of data processing calculations, and then enabled the importance of the direction of force application to early acceleration sprint performance to be established. Firstly, the RF- $\mathrm{v}_{H}$ profile over the whole acceleration phase was shown to differ between using step-averaged RF data calculated from either instantaneous RF data or step-averaged force data, and thus hypothesis one was accepted. Secondly, using the step-averaged force data to determine RF, average RF during early acceleration was shown to be of greater importance for early acceleration performance than average absolute GRF magnitude, particularly for block starts, and hypothesis two was also accepted. Thirdly, $\mathrm{RF}_{0}$ was shown to be around 1.3-1.4 times more important for early acceleration performance than $\mathrm{D}_{\mathrm{RF}}$ (both determined during just early acceleration), and thus hypothesis three was rejected. Finally, when using the outputs obtained from a simple macroscopic model fitted to the $\mathrm{V}_{\mathrm{H}}$-time data over the entire acceleration phase, there was a nearly perfect relationship between $\mathrm{RF}_{\mathrm{MAX}}$ and early acceleration performance from both block and standing starts.

The effect of different input data for determining step-averaged ratio of forces

For all participants from both standing and block starts, there was a lower $y$-intercept $\left(\mathrm{RF}_{0}\right.$ FP_all $)$ and a shallower gradient ( $\left.D_{\text {RF-FP_all }}\right)$ when the step-averaged RF values were obtained 
from instantaneous RF data compared with from step-averaged force data (Table 1; Figures 1-2). Whilst the relationship between the output values from the two calculation methods was very large (all $r>0.74$ ), this non-perfect relationship reflects that there was clearly not just a systematic effect of the different calculation methods (Figure 1). The random difference between the two methods ( $95 \%$ limits of agreement $= \pm 0.4$ and $\pm 0.6 \% \cdot \mathrm{s} / \mathrm{m}$ for $\mathrm{D}_{\mathrm{RF}-\mathrm{FP}}$ all for block and standing starts, respectively; \pm 3.3 and $\pm 4.3 \%$ for $\mathrm{RF}_{0-\mathrm{FP} \_ \text {all }}$ for block and standing starts, respectively) reveals that the effects of these two calculation methods vary from athlete to athlete. This occurs because of individual differences in the shape of the instantaneous RF time-history, particularly during late stance when the magnitude of force is relatively low and instantaneous RF can reach high values (Figure 6). When used to determine average RF across stance, these late stance instantaneous RF values have an equal weighting to all other timepoints during stance, despite occurring when the horizontal component of the GRF is low and decreasing further (i.e. it is less "functionally effective"). The effects of this increase in averaged instantaneous RF during late stance on the $\mathrm{RF}-\mathrm{v}_{\mathrm{H}}$ relationship consistently becomes more pronounced as the acceleration phase progresses (Figure 2; Figures A1a-d). This may in part be due to the average RF during the early-mid part of stance being higher during early acceleration and thus the whole-stance-averaged values are less affected by the higher values towards toe-off than they are later in the acceleration phase. The rate of decline in the horizontal component of the GRF also becomes relatively lower than that of the vertical component as the acceleration phase progresses (Figure 6). These differences in the shapes of the GRF profile during late stance, which are likely due to different stance leg joint kinematics during late stance as the acceleration phase progresses (Schache et al., 2019), may also partly explain the observed differences. Although it is not possible to determine the exact reasons from the current analysis, this effect clearly causes the step-averaged RF values from the method which used the instantaneous RF data to become increasingly higher than those from the method which used the step-averaged force data as the acceleration phase progresses (Figure 2; Figures A1a-d). The use of step-averaged RF values from the step-averaged force data provides data closer to the mechanical reality of step-averaged 
acceleration approaching zero (aside from the need to overcome air resistance) towards the end of the acceleration phase. In contrast, although more appropriate as a direct measure of mean RF during stance, the mechanical meaning and applied interpretation of the average of the instantaneous RF data is less clear.
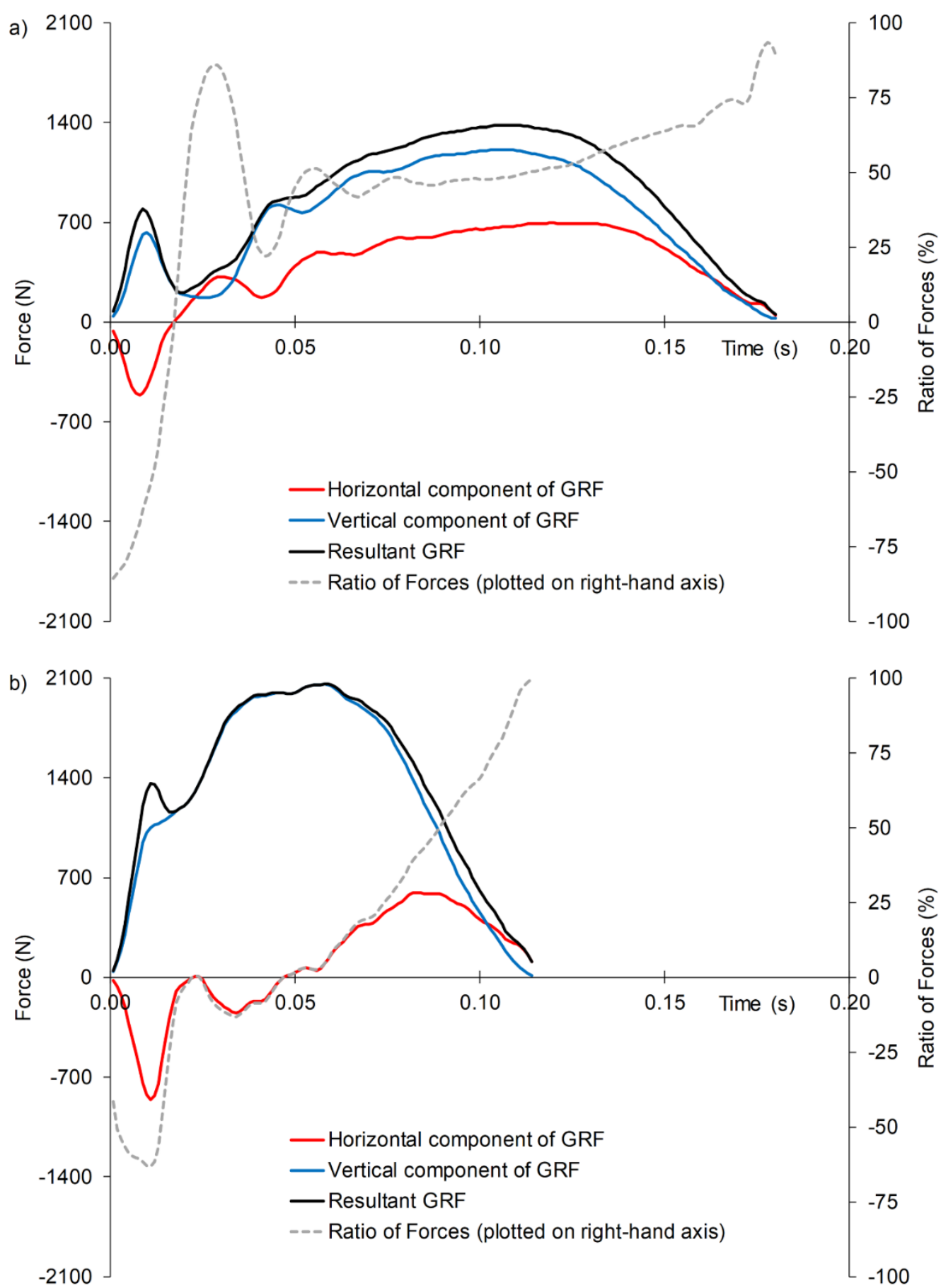

Figure 6. Vertical and horizontal components of ground reaction force, and instantaneous ratio of forces (plotted on secondary y-axis) during a) step 1 and b) step 13 for a typical trial from blocks. 
Given that the $\mathrm{RF}-\mathrm{v}_{\mathrm{H}}$ relationship clearly differs between the two different methods for calculating step-averaged $R F$, a direct comparison of $R F-v_{H}$ metrics (e.g. $R F_{0}$ or $R_{M A X}, D_{R F}$ ) between studies which have used different calculation methods is not appropriate. Mathematically, these differences occur because of a difference in when values are squared and averaged between the two calculation methods. Care must therefore be taken to understand which calculation method has been employed when interpreting the results of previous studies. Whilst there is a systematic difference between the two methods, there is also considerable random error due to individual differences and a simple offset may not be appropriate (Figure 1). It is recommended in future that the step-averaged force data are used when step-averaged RF values are desired because these appear to be less affected by extreme RF values at the latter end of the stance phase where force magnitudes are low (particularly evident at higher speeds). These also appear closer to the mechanical reality and likely provide a more appropriate assessment of the "mechanical effectiveness" over the entire step. One important consideration of this calculation approach is that it is perhaps more appropriate to be considered a ratio of impulses rather than a ratio of forces given that stepaveraged forces are used. Furthermore, it must be noted that the use of the mean of the instantaneous horizontal and vertical force components to determine the mean resultant force when using step-averaged force data will not always yield the same value as the mean of the instantaneous resultant forces (mean \pm SD difference in step averaged RF over all steps in the current data $=0.51 \pm 0.37 \%$ ), and this should also be clearly identified. Finally, the use of instantaneous RF data may still be of value where within-stance technique is of interest, such as when investigating the shape of the RF curve over a block exit or stance phase (e.g. Bezodis et al., 2019; Colyer et al., 2018). However, in such instances, researchers and practitioners must be cautious of over-interpreting instantaneous RF values when absolute magnitudes of force are low. As a minimum, it is recommended that all studies report their RF calculation methods in sufficient detail such that readers can identify exactly how stepaveraged RF was determined, as this has not always been clear in studies published to date. 
The importance of RF versus absolute GRF magnitude for early acceleration performance

Having identified that the use of step-averaged force data provided more appropriate measures of step-averaged RF, this calculation approach was used in all subsequent reported analyses. There was a very large relationship between the ability to produce a high average RF during early acceleration and early acceleration performance from both block $(r=0.88)$ and standing $(r=0.84)$ starts $^{4}$. There was a large relationship between average RF and average GRF magnitude during early acceleration $(r=0.59$ and 0.63 for block and standing starts, respectively). This relationship may be a function of the sample of sprinters studied in which some of the athletes with a high GRF are also those with a high RF but, intuitively, these are two independent concepts and there clearly remains considerable variation in RF which is not explained by the resultant GRF magnitude. The magnitude of resultant force applied and the direction of application of this force are therefore different qualities during early acceleration, much like when considered across the entire acceleration phase (Rabita et al., 2015). When assessing the relative importance of these two qualities during early acceleration, RF was found to be of considerably greater importance than resultant GRF magnitude over block exit and the first four steps from block starts (i.e. an 8-fold greater $\beta$ coefficient), but they were of more similar importance over the initial push-off and first four steps from standing starts (i.e. a 1.4-fold greater $\beta$-coefficient for RF compared with resultant GRF magnitude). Although the importance of the direction of force application has been widely established across an entire acceleration phase (i.e. up to maximum velocity; Morin et al., 2011; Rabita et al., 2015), this confirms that it remains a highly important ability when just early acceleration is of interest.

\footnotetext{
${ }^{4}$ In further support of the recommendation to adopt the use of the step-averaged force method for determining step-averaged RF, it is worthy of note that if the step-averaged RF data obtained using the instantaneous RF data are used in these correlations, the corresponding correlation coefficients with NAHEP drop to 0.61 and 0.62 (from 0.88 and 0.84 ; see Appendix, Table A1). Importantly for applied practice, the step-averaged RF data obtained from step-averaged force data therefore also correspond more strongly to performance.
} 
The importance of $R F_{0}$ and $D_{R F}$ for early acceleration performance

When combined in a multiple linear regression across the group, $R F_{0-F P}$ was found to be of greater relative importance for performance than $D_{\text {RF-FP }}$ (Table 2). Although they may each reflect independent qualities, it must be considered that when they are calculated in the current way, $\mathrm{RF}_{0-\mathrm{FP}}$ and $\mathrm{D}_{\mathrm{RF}-\mathrm{FP}}$ are both measures of a trendline which is ultimately dependent on the five data points they are fitted to (i.e. initial push-off/block exit and each of the first four steps), but that they can provide separate measures of the RF magnitude and of its rate of decline. $\mathrm{RF}_{0-\mathrm{FP}}$ and $\mathrm{D}_{\mathrm{RF}-\mathrm{FP}}$ combined to explain $93 \%$ (block starts) or $95 \%$ (standing starts) of the variance in early acceleration performance (Table 2), which is well illustrated by the fact that the datapoints lie very close to a $3 \mathrm{D}$ plane with $\mathrm{RF}_{0-\mathrm{FP}}$ and $\mathrm{D}_{\mathrm{RF}-\mathrm{FP}}$ as the predictor variables and NAHEP as the dependent variable (Figure 5; Figure S1). This suggests that whilst having a high $R F_{0-F P}$ is of primary concern for practitioners, having a less rapid decline in $R F\left(D_{R F-F P}\right)$, even just during this early part of the acceleration phase, is then an important feature which

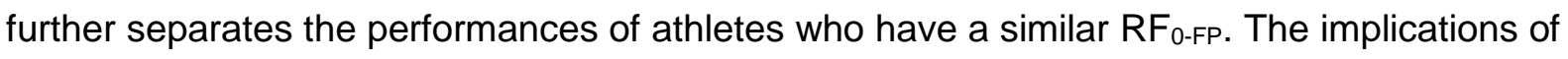
this for early acceleration performance appear to be that athletes should initially prioritise achieving high RF values rather than preventing the subsequent drop off in RF. There appears to be less benefit of having a less rapid initial decline in RF for athletes who are incapable of starting from high RF values, but once athletes are capable of achieving high initial RF values, working to maintain these high RF values as the early steps of a sprint progress is clearly of value for performance. However, these findings are based on a cross-sectional observation and future experimental work is required to investigate the relative trainability of an athlete's RF- $\mathrm{v}_{\mathrm{H}}$ profile.

Relationships between simple macroscopic model outputs and early acceleration performance The $\mathrm{RF}_{\mathrm{MAX}-\mathrm{M}}$ measure obtained from a simple macroscopic model (Samozino et al., 2016) fitted to the $\mathrm{v}_{\mathrm{H}}$-t profile over the entire acceleration phase provided a near perfect predictor of performance over the initial acceleration phase from both block and standing starts (Table 3). Performance was quantified as the average external power produced over the initial push- 
off/block exit and the first four steps because this provides an objective measure of early acceleration performance (Bezodis et al., 2010). It is therefore logical that RF $F_{\text {AXX-M }}$ correlates highly with performance because $\mathrm{RF}_{\mathrm{MAX}}$ correlates highly with maximum modelled power (' $\mathrm{P}_{\mathrm{MAX}}$ ') given the simple nature of the mono-exponential fit to the $\mathrm{v}_{\mathrm{H}}$-t profile (Haugen et al., 2019b). Although this mono-exponential model is a simple representation of reality which does not account for the energy required to move the limbs about the $\mathrm{CM}$, to raise the $\mathrm{CM}$, or to overcome the effects of braking (Pavei et al., 2019), the ability to translate the CM over as great a horizontal displacement as possible in as short a time as possible is the performance requirement in accelerative sprinting. Therefore, although 'horizontal power' is not a real quantity in a strict mechanical sense (Vigotsky et al., 2019), the relevance of this quasi-power quantity to sprint acceleration performance is clear, and the simplicity of determining it from the mono-exponential model, combined with how closely this aligns with average 'power' over multiple steps during early acceleration, provides clear and relevant applications to practice.

These simple macroscopic model findings reiterate the importance of athletes being able to achieve high RF values during the early part of a sprint but are also potentially valuable for applied practitioners since $R F_{\text {MAX }}$, which is proposed to approximately correspond to the $R F$ value of the first step, can be relatively readily obtained in the field. However, it must be noted that the data which the macroscopic model was fitted to in the current study came from a 'gold standard' force platform system. Whilst highly similar modelled $\mathrm{v}_{\mathrm{H}}$-t profiles can be obtained from other measurement systems such as lasers (Morin et al., 2019), there are differences in the profiles compared with those obtained from force platforms and thus the predictive capabilities could be slightly different.

\section{Reflections, limitations and future directions}

This study directly measured the ground reaction forces over the entire acceleration phase from a large group of trained sprinters commencing from both block and standing starts. The data therefore exhibited high levels of both internal and ecological validity and thus provided 
an appropriate dataset to address both methodological and applied issues relating to the understanding of the importance of RF during early acceleration. Despite all being sprint trained, none of the current cohort could be considered to be competitive at the very highest level and thus the application of the current findings to truly elite sprinters or other populations remains unknown. Some standing start trials were excluded from the current analysis because of a lack of confidence in the automatically determined movement onset. Whilst this was not an issue for the current aims regarding the determination of the importance of RF (particularly given the relatively large participant numbers), future research is warranted to investigate the objective determination of movement onset, particularly during standing starts. This is particularly important for when other, non-'gold-standard' measurement technologies are used, and may also be of value in helping to inform the appropriateness of both the time delay used in the fitting of the simple macroscopic model (Samozino, 2018) and the $0.3 \mathrm{~s}$ offset used in the extraction of RF measures from it (Samozino et al., 2016). Furthermore, only simple linear functions were fitted to the RF- $\mathrm{v}_{\mathrm{H}}$ data during early acceleration, and visual analysis of some trials (see Appendix, Figure A1a-d) suggested that this may not always be the most appropriate fit. Other higher order functions, or combinations of linear functions either side of a breakpoint (e.g. Nagahara et al., 2014; 2020; von Lieres und Wilkau, 2020) are worthy of future exploration to assess whether different descriptions of the RF- $\mathrm{v}_{\mathrm{H}}$ profile during early acceleration provide any alternative information which is relevant to early acceleration performance.

\section{Conclusions, practical applications and recommendations}

There are several important points which have arisen from this study and are relevant to researchers and/or practitioners interested in the importance of ratio of forces for (early) acceleration:

1. The use of different data processing methods for obtaining step-averaged RF values affects the features of the obtained RF- $\mathrm{V}_{\mathrm{H}}$ relationship, and these differences are not systematic across all individuals. It is recommended that step-averaged force data are 
used to obtain step-averaged RF values because these appear to be less affected by extreme RF values at the latter end of stance and they also appear to relate more closely to the true mechanical reality. There may still be merit in investigating instantaneous RF data for certain applications, and researchers are encouraged to report their RF calculations in full to enable readers to easily understand the approach they have adopted.

2. The ability to direct force horizontally is important for early acceleration, with its importance outweighing that of the magnitude of force applied, particularly for trials commencing from starting blocks.

3. It appears that athletes should firstly prioritise achieving high initial RF values in order to achieve high levels of early acceleration performance. Once they are capable of this, aiming to prevent the drop off in RF during early acceleration then becomes of benefit.

4. RF $F_{\text {MAX }}$ from a simple macroscopic model provides a near perfect predictor of early acceleration performance and further supports the importance of being able to achieve high initial RF values during early acceleration. The fact that this measure from a simple model of the whole acceleration phase appears to be the strongest single predictor indicator of early acceleration performance (i.e. better than any RF metric obtained directly from just the early acceleration phase) suggests that some of the more subtle variation in RF profiles during early acceleration may not be highly relevant to performance. Given the importance of modelled $\mathrm{RF}_{\mathrm{MAX}}$ from the entire acceleration phase for early acceleration performance, practitioners could use $\mathrm{RF}_{\mathrm{MAX}}$ as an early acceleration performance measure in field-based testing scenarios. However, caution is still needed as further evaluations are required using inputs from non-force platform data and across other populations.

5. Whilst there were some small differences in the magnitudes of the effects between starts from blocks or standing, all of the above applications and recommendations are relevant to both types of start. Care should be taken to ensure that the initiation of 
movement occurs in one smooth action (predominantly for standing starts) for both research and practice purposes to avoid movement onset issues which can influence the properties of the determined RF abilities during early acceleration. 


\section{References}

Bayne, H. (2018). Force-velocity-power profiles of elite sprinters: inter- and intra-individual determinants of performance. ISBS Proceedings Archive, 36(1), Article 245. Retrieved from https://commons.nmu.edu/isbs/vol36/iss1/245.

Bezodis, N. E, Salo, A. I. T, \& Trewartha, G. (2010). Choice of sprint start performance measure affects the performance-based ranking within a group of sprinters: which is the most appropriate measure? Sports Biomechanics, 9(4), 258-269.

Bezodis, N. E., Walton, S. P., \& Nagahara, R. (2019). Understanding the track and field sprint start through a functional analysis of the external force features which contribute to higher levels of block phase performance. Journal of Sports Sciences, 37(5), 560-567.

Colyer, S. L., Nagahara, R. \& Salo, A. I. T. (2018). Kinetic demands of sprinting shift across the acceleration phase: novel analysis of entire force waveforms. Scandinavian Journal of Medicine and Science in Sports, 28(7), 1784-1792.

Haugen, T., McGhie, D. \& Ettema, G. (2019a). Sprint running: from fundamental mechanics to practice - a review. European Journal of Applied Physiology, 119(6), 1273-1287.

Haugen, T. A., Breitschädel, F. \& Seiler. (2019b). Sprint mechanical variables in elite athletes: are force-velocity profiles sport specific or individual? Plos One, 14(7), e0215551.

Hopkins, W. G. (2014). A scale of magnitudes for effect statistics. Retrieved from http://www.sportsci.org/resource/stats/.

Morin, J.-B., Edouard, P., \& Samozino, P. (2011). Technical ability of force application as a determinant factor of sprint performance. Medicine \& Science in Sports \& Exercise, 43(9), 1680-1688

Morin, J.-B., Bourdin, M., Edouard, P., Peyrot, N., Samozino, P. \& Lacour, J.-R. (2012). Mechanical determinants of $100-\mathrm{m}$ sprint running performance. European Journal of Applied Physiology, 112(11), 3921-3930. 
Morin, J.-B., Samozino, P., Murata, M., Cross, M. R. \& Nagahara, R. (2019). A simple method for computing sprint acceleration kinetics from running velocity data: replication study with improved design. Journal of Biomechanics, 94, 82-87.

Nagahara, R., Matsubayashi, T., Matsuo, A. \& Zushi, K. (2014). Kinematics of transition during human accelerated sprinting. Biology Open, 3, 689-699.

Nagahara, R., Kanehisa, H. \& Fukunaga, T. (2020). Ground reaction force across the transition during sprint acceleration. Scandinavian Journal of Medicine and Science in Sports, 30(3), 450-461.

Pavei, G., Zamparo, P., Fujii, N., Otsu, T., Numazu, N., Minetti, A. E., \& Monte, A. (2019). Comprehensive mechanical power analysis in sprint running acceleration. Scandinavian Journal of Medicine and Science in Sports, 29(12), 1892-1900.

Rabita, G., Dorel, S., Slawinski, J., Saez-de-Villarreal, E., Couturier, A., Samozino, P. \& Morin J.-B. (2015). Sprint mechanics in world-class athletes: a new insight into the limits of human locomotion. Scandinavian Journal of Medicine and Science in Sports, 25(5), 583-594.

Samozino, P. (2018). A simple method for measuring force, velocity and power capabilities and mechanical effectiveness during sprint running. In J.-B. Morin \& P. Samozino (Eds), Biomechanics of Training and Testing (pp. 237-267). Cham, Switzerland: Springer International Publishing.

Samozino, P., Rabita, G., Dorel, S., Slawinski, J., Peyrot, N., Saez de Villarreal, E. \& Morin, J.-B. (2016). A simple method for measuring power, force, velocity properties, and mechanical effectiveness in sprint running. Scandinavian Journal of Medicine and Science in Sports, 26(6), 648-658.

Schache, A. G., Lai, A. K. M., Brown, N. A. T., Crossley, K. M. \& Pandy, M. G. (2019). Lowerlimb joint mechanics during maximum acceleration sprinting. Journal of Experimental Biology, 222(22), jeb209460. 
Vigotsky, A. D., Zelik, K. E., Lake, J., \& Hinrichs, R. N. (2019). Mechanical misconceptions: have we lost the "mechanics" in "sports biomechanics"? Journal of Biomechanics, 93, $1-5$.

von Lieres und Wilkau, H. C., Irwin, G., Bezodis, N. E., Simpson, S., \& Bezodis, I. N. (2020). Phase analysis in maximal sprinting: an investigation of step-to-step technical changes between the initial acceleration, transition and maximal velocity phases. Sports Biomechanics, 19(2), 141-156.

Winter, D. A. (2005) Biomechanics and motor control of human movement (3rd ed.). New York, NY: Wiley. 


\section{Appendix}

Table A1. Pearson's correlations $(r)$ between average RF (RF $\left.F_{M E A N-F P}\right)$ and performance (NAHEP) during early acceleration, using different combinations of input data.

\begin{tabular}{|c|c|c|c|}
\hline & Measure & Blocks & Standing \\
\hline \multirow{3}{*}{$\begin{array}{c}\text { FP data from push-off/blocks and first four steps } \\
\text { (using step-averaged RF values from step-averaged } \\
\text { force data) } \\
\text { (i.e. data used in main results section) }\end{array}$} & $\mathrm{RF}_{\mathrm{MEAN}-\mathrm{FP}}$ & $0.88^{* * *}$ & $0.84^{\star * *}$ \\
\hline & $R F_{0-F P}$ & $0.59^{\star *}$ & $0.79^{\star * \star}$ \\
\hline & $D_{\text {RF-FP }}$ & -0.04 & $-0.55^{\star *}$ \\
\hline \multirow{3}{*}{$\begin{array}{l}\text { FP data from push-off/blocks and first four steps } \\
\text { (using step-averaged RF values from instantaneous RF } \\
\text { data) }\end{array}$} & $\mathrm{RF}_{\mathrm{MEAN}-\mathrm{FP}}$ & $0.61^{\star *}$ & $0.62^{\star *}$ \\
\hline & $\mathrm{RF}_{0-\mathrm{FP}}$ & 0.38 & $0.76^{\star \star \star}$ \\
\hline & $D_{\text {RF-FP }}$ & 0.01 & $-0.62^{* *}$ \\
\hline \multirow{3}{*}{$\begin{array}{l}\text { FP data from first four steps (excluding push-off/blocks) } \\
\text { (using step-averaged RF values from step-averaged } \\
\text { force data) }\end{array}$} & $\mathrm{RF}_{\mathrm{MEAN}-\mathrm{FP}}$ & $0.80^{\star \star *}$ & $0.61^{* *}$ \\
\hline & $\mathrm{RF}_{0-\mathrm{FP}}$ & 0.38 & 0.20 \\
\hline & $D_{\text {RF-FP }}$ & -0.09 & 0.02 \\
\hline \multirow{3}{*}{$\begin{array}{l}\text { FP data from first four steps (excluding push-off/blocks) } \\
\text { (using step-averaged RF values from instantaneous RF } \\
\text { data) }\end{array}$} & $\mathrm{RF}_{\mathrm{MEAN}-\mathrm{FP}}$ & $0.51^{*}$ & 0.17 \\
\hline & $\mathrm{RF}_{0-\mathrm{FP}}$ & 0.24 & -0.03 \\
\hline & $D_{\text {RF-FP }}$ & -0.09 & 0.12 \\
\hline
\end{tabular}

${ }^{*} \mathrm{p}<0.05 ;{ }^{* \star} \mathrm{p}<0.01 ;{ }^{* \star *} \mathrm{p}<0.001$ 
Table A2. Pearson's correlations ( $r$ ) between the outputs from the RF- $v_{H}$ data when obtained with either the step averaged force data to obtain RF or the instantaneous RF data to obtain RF. Different combinations of input steps are used in the linear fit of the RF- $\mathrm{v}_{H}$ profile (i.e. entire acceleration phase, early acceleration only, initial push-off/block exit included or excluded). The first two rows (in bold) correspond to the data presented in Figure 1 and the start of the second paragraph of results text in the manuscript (i.e. $\mathrm{RF}_{0-\mathrm{FP} \_a l l}$ and $\mathrm{D}_{\mathrm{RF}-\mathrm{FP} \_a l l}$ ).

\begin{tabular}{|c|c|c|}
\hline Output variable from the step averaged $R F-v_{H}$ data & Standing & Blocks \\
\hline $\mathbf{R F}_{0}$ over push-off/block exit and all steps $\left(\mathrm{RF}_{0-\mathrm{FP} \_ \text {all }}\right)$ & $0.82^{\star \star \star}$ & $0.85^{\star \star \star}$ \\
\hline$D_{R F}$ over push-off/block exit and all steps ( $D_{\text {RF-FP_all }}$ ) & $0.75^{\star \star *}$ & $0.88^{\star * *}$ \\
\hline $\mathrm{RF}_{0}$ over all steps excluding push-off/block exit & $0.48^{*}$ & $0.79^{* \star *}$ \\
\hline$D_{\mathrm{RF}}$ over all steps excluding push-off/block exit & $0.50^{*}$ & $0.82^{* * *}$ \\
\hline Average RF over first 4 steps and push-off/block exit & $0.62^{* \star}$ & $0.80^{* * *}$ \\
\hline Average RF over first 4 steps excluding push-off/block exit & $0.44^{*}$ & $0.80^{* * *}$ \\
\hline $\mathrm{RF}_{0}$ over first 4 steps and push-off/block exit & $0.98^{\star \star *}$ & $0.86^{\star \star \star}$ \\
\hline $\mathrm{RF}_{0}$ over first 4 steps excluding push-off/block exit & $0.80^{* * *}$ & $0.86^{\star * *}$ \\
\hline$D_{\mathrm{RF}}$ over first 4 steps and push-off/block exit & $0.96^{* * *}$ & $0.88^{* * *}$ \\
\hline$D_{\mathrm{RF}}$ over first 4 steps excluding push-off/block exit & $0.84^{* \star *}$ & $0.85^{\star \star *}$ \\
\hline
\end{tabular}


Table A3. Pearson's correlations ( $r$ ) between force platform determined (from the entire acceleration phase) and simple macroscopic model RF variables for block and standing starts.

\begin{tabular}{|c|c|c|c|c|c|c|c|}
\hline \multirow[t]{2}{*}{ Blocks } & & \multicolumn{2}{|c|}{$\begin{array}{c}\text { Simple macroscopic } \\
\text { model }\end{array}$} & \multirow{2}{*}{\multicolumn{2}{|c|}{ Standing }} & \multicolumn{2}{|c|}{$\begin{array}{c}\text { Simple macroscopic } \\
\text { model }\end{array}$} \\
\hline & & $\mathrm{RF}_{\mathrm{MAX}-\mathrm{M}}$ & $\mathrm{D}_{\mathrm{RF}-\mathrm{M}}$ & & & $\mathrm{RF}_{\mathrm{MAX}-\mathrm{M}}$ & $\mathrm{D}_{\mathrm{RF}-\mathrm{M}}$ \\
\hline 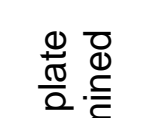 & $\begin{array}{l}\mathrm{RF}_{0-} \\
\mathrm{FP}\end{array}$ & $0.93^{\star \star \star}$ & $\left(-0.84^{\star \star \star}\right)$ & 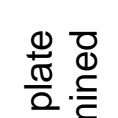 & $\mathrm{RF}_{0-\mathrm{FP}}$ & $0.88^{\star \star \star}$ & $\left(-0.91^{\star \star *}\right)$ \\
\hline 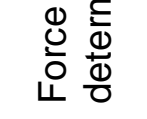 & $\begin{array}{c}D_{\text {RF- }} \\
F P\end{array}$ & $\left(-0.56^{\star \star}\right)$ & $0.98^{\star \star \star}$ & 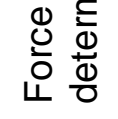 & $D_{\text {RF-FP }}$ & $\left(-0.59^{\star \star}\right)$ & $0.86^{\star \star \star}$ \\
\hline
\end{tabular}

${ }^{*} p<0.05 ;{ }^{* *} p<0.01 ;{ }^{* * *} p<0.001$ 
Figures A1 (a-d) below are presented to illustrate examples of RF- $\mathrm{v}_{\mathrm{H}}$ datapoints (all from block starts) where simple linear fits during early acceleration may not be appropriate (a and b) or where a linear fit may be appropriate if the initial push-off/block exit is excluded, but early acceleration is clearly different from the remainder of the sprint (c and d). Solid trendlines correspond to the first 5 data points, dashed trendlines correspond to all data points.

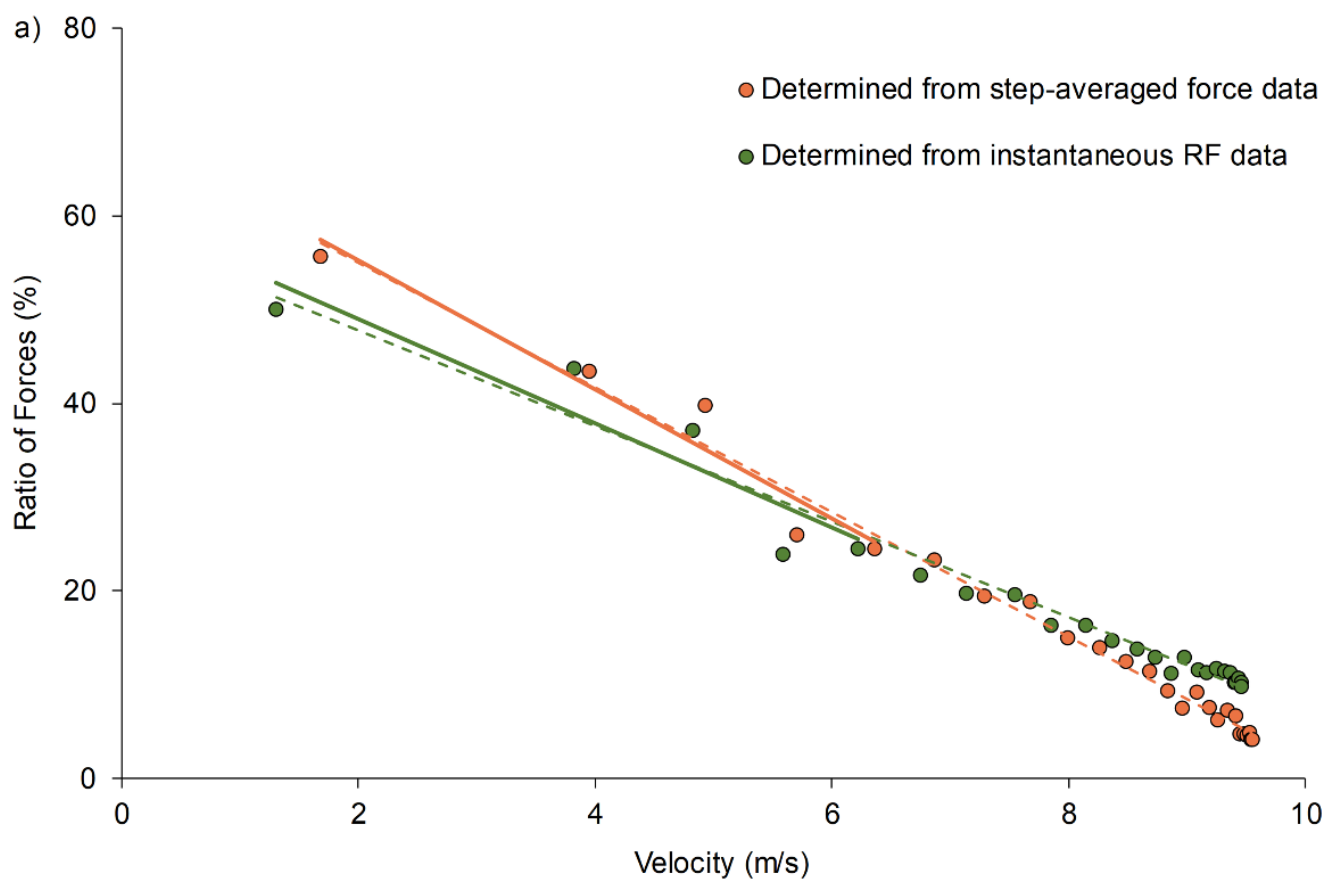

Figure A1a. RF is higher during block exit and first two steps compared with the remainder of the acceleration phase (P12T06)

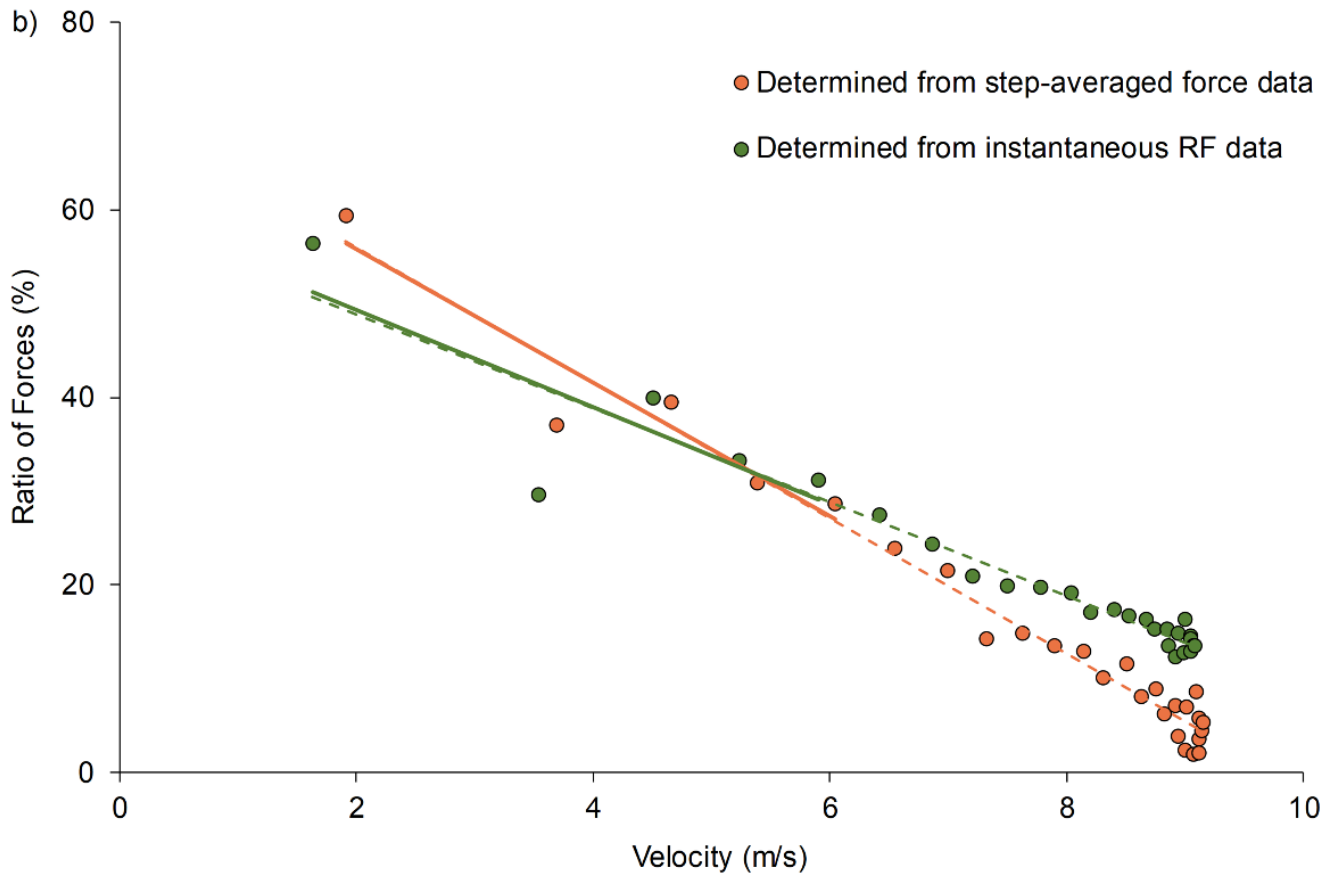

Figure A1b. RF is lower in the first step after block exit compared with block exit and the remainder of the acceleration phase (P14T13). 


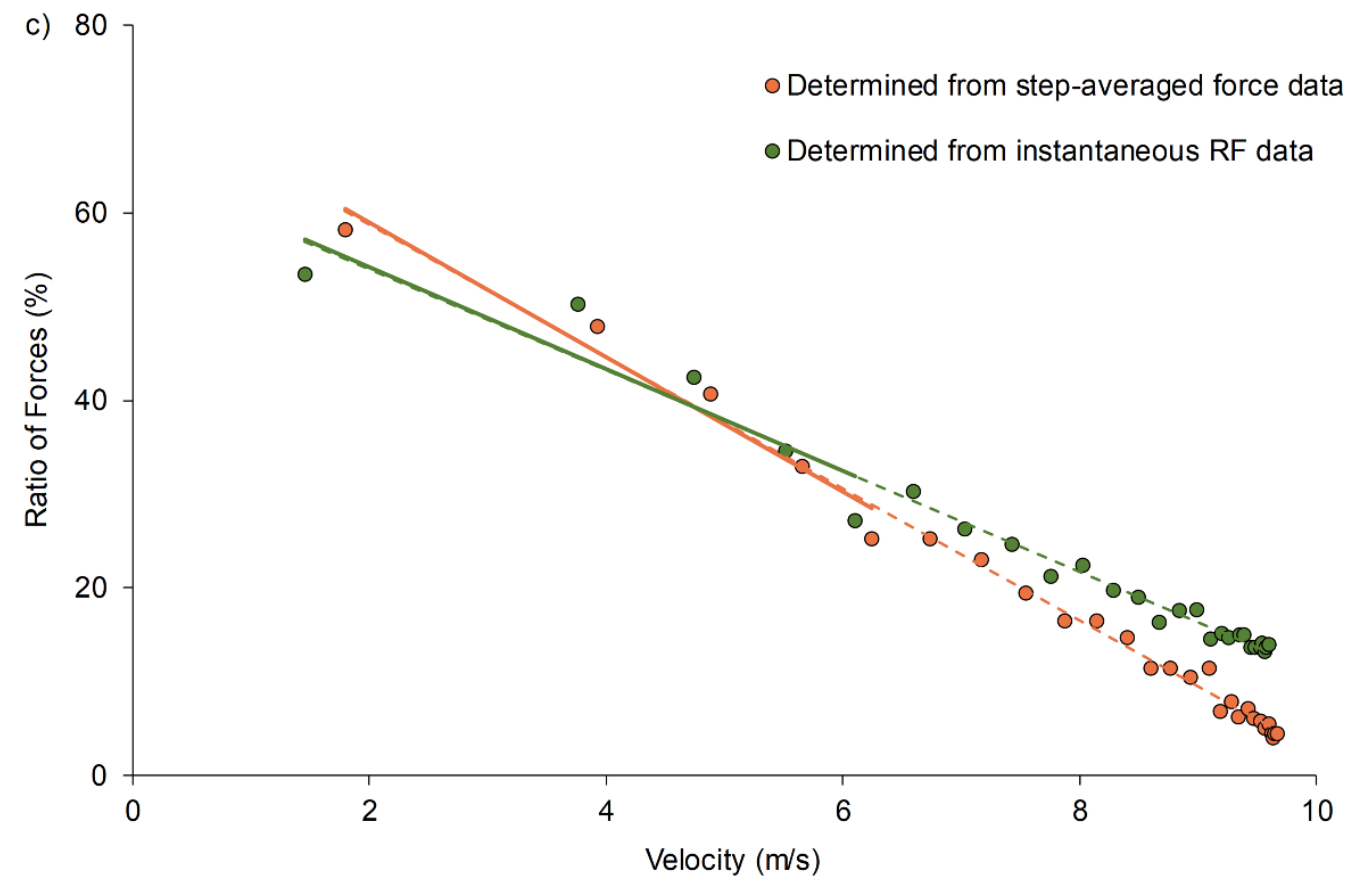

Figure A1c. RF shows a different relationship during the first four steps on the track compared with the remainder of the acceleration phase, and compared with block exit (P22T19).

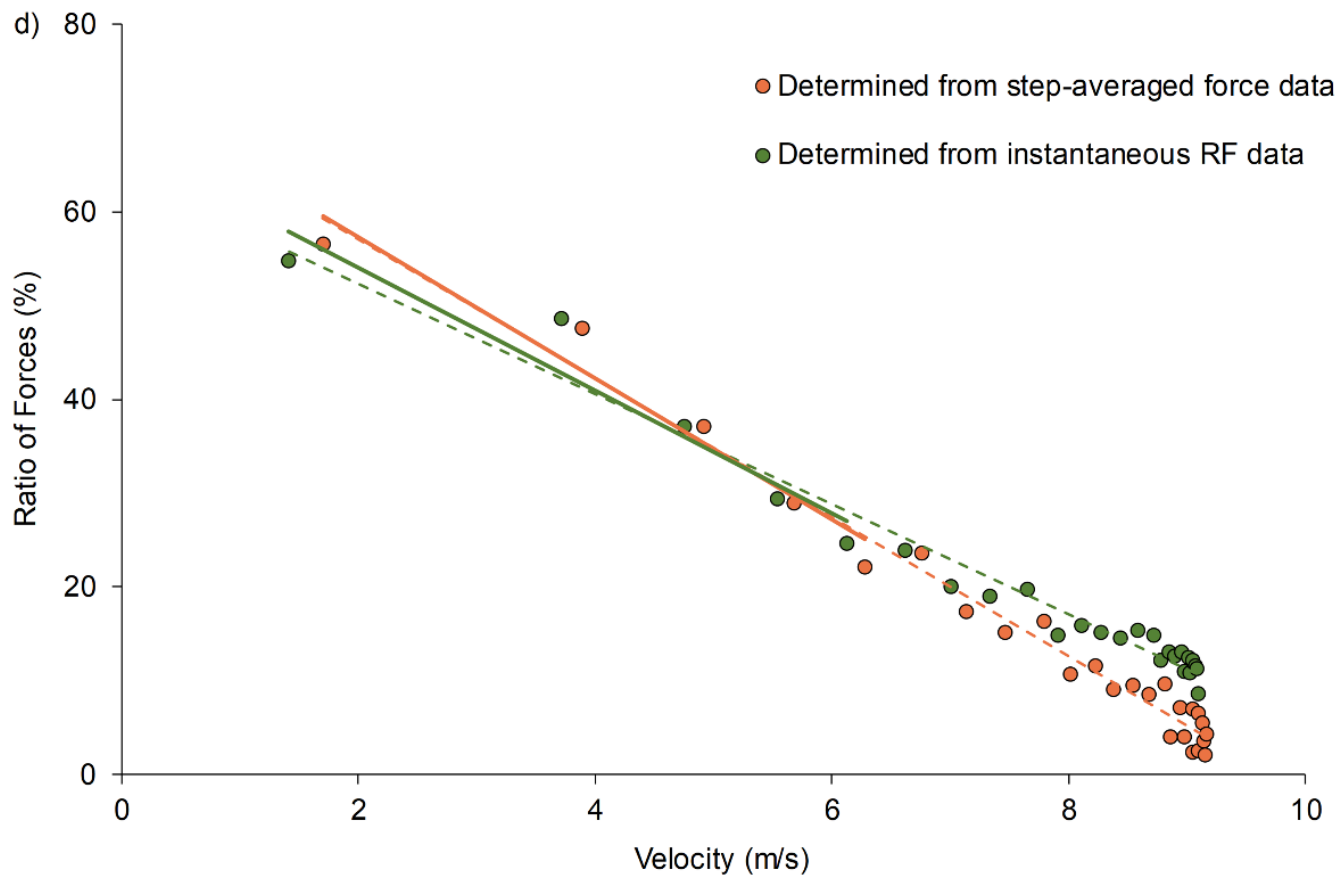

Figure A1d. RF shows a different relationship during the first four steps on the track compared with the remainder of the acceleration phase, and compared with block exit (P16T27). 